\title{
Wavelets From Laguerre Polynomials AND TOEPLITZ-TYPE OPERATORS
}

\author{
Ondrej HUTNÍK1
}

\begin{abstract}
We study Toeplitz-type operators with respect to specific wavelets whose Fourier transforms are related to Laguerre polynomials. On the one hand, this choice of wavelets underlines the fact that these operators acting on wavelet subspaces share many properties with the classical Toeplitz operators acting on the Bergman spaces. On the other hand, it enables to study poly-Bergman spaces and Toeplitz operators acting on them from a different perspective. Restricting to symbols depending only on vertical variable in the upper half-plane of the complex plane these operators are unitarily equivalent to a multiplication operator with a certain function. Since this function is responsible for many interesting features of these Toeplitz-type operators and their algebras, we investigate its behavior in more detail. As a by-product we obtain an interesting observation about the asymptotic behavior of true polyanalytic Bergman spaces. Isomorphisms between the CalderónToeplitz operator algebras and functional algebras are described and their consequences are discussed.
\end{abstract}

\section{Introduction}

The structure of the space of wavelet transforms inside $L_{2}(G, \mathrm{~d} \nu)$ (the space of all square-integrable functions on the affine group $G$ with respect to the left invariant Haar measure $\mathrm{d} \nu$ ) was described in our paper 9. The key tool in this description is the (Bargmann-type) transform giving an isometrical isomorphism of the space $L_{2}(G, \mathrm{~d} \nu)$ under which the space of wavelet transforms is mapped onto tensor product of $L_{2}$-spaces where one of them is the rank-one space generated by a suitable function. This isomorphism is constructed according to the general Vasilevski scheme of decomposition of Hilbert spaces presented in 25. which was systematically applied in his book [31, and provides an interesting tool to study Toeplitz-type operators based on the Calderón reproducing formula and acting on wavelet subspaces. This idea was developed in general setting in paper [10] where some results on Toeplitz-type operators related to wavelets were also given. Then this technique was used in [1] for fundamental case where the wavelet subspaces arise from functions whose Fourier transforms are related to Laguerre polynomials. Note that in this case the rank-one space is generated by Laguerre functions $\ell_{k}(x)=\mathrm{e}^{-x / 2} L_{k}(x)$ forming an orthonormal basis in $L_{2}\left(\mathbb{R}_{+}\right)$, where $L_{k}(x)$ stands for the Laguerre polynomial of degree $k$ and type 0 at $x$.

This paper is a further continuation of the above mentioned research. Here we are interested in some properties of these Toeplitz-type operators acting on wavelet subspaces in detail. In accordance with paper [1] we consider specific wavelets which enable us to obtain an interesting generalization of the classical

\footnotetext{
${ }^{1}$ Mathematics Subject Classification (2010): Primary 47B35, 42C40, Secondary 47G30, $47 \mathrm{~L} 80$

Key words and phrases: Wavelet, admissibility condition, continuous wavelet transform, Calderón reproducing formula, Toeplitz operator, Laguerre polynomial, operator algebra, improper parameter-dependent integral
} 
case of Toeplitz operators acting on the Bergman space. This choice of wavelets also underlines the intriguing patterns which the corresponding wavelet subspaces and poly-Bergman spaces share. In fact, the main source of motivation for our study comes from the Vasilevski book [31] summarizing results of author and his collaborators on classical Toeplitz operators and their algebras on the Bergman space. The presented methods and techniques in that book are very useful and inspiring for our purposes. Thus, on the one hand, many obtained results of this paper as well as paper 12 may be simply viewed as "wavelet analogs" of results known for the classical Toeplitz operators. On the other hand, the described generalization of Toeplitz operators goes by another direction as in the case of Toeplitz operators acting on weighted Bergman spaces, and gives rise to many new and interesting questions. The organization of this paper is as follows.

Section 2; Representation of wavelet subspaces We introduce basic notions and basic tools of our study including a parameterized family of wavelets $\left\{\psi^{(k)}\right\}_{k \in \mathbb{Z}_{+}}$whose Fourier transform is related to Laguerre polynomials $L_{k}(x)$. Then we introduce the isometrical isomorphism

$$
U: L_{2}(G, \mathrm{~d} \nu) \rightarrow L_{2}(\mathbb{R}) \otimes L_{2}\left(\mathbb{R}_{+}\right)
$$

describing the structure of wavelet subspaces $A^{(k)}$ (related to wavelet $\psi^{(k)}$ and Hardy-space functions) inside $L_{2}(G, \mathrm{~d} \nu)$. This result also allows us to describe the direct and natural connection between wavelet subspaces and Hardy spaces. Further, we construct operators $R_{k}$ and $R_{k}^{*}$ providing decomposition of orthogonal projection $P^{(k)}$ from $L_{2}(G, \mathrm{~d} \nu)$ onto wavelet subspace $A^{(k)}$ and of identity operator $I$ on $L_{2}\left(\mathbb{R}_{+}\right)$. Note that the operator $R_{k}$ is an exact analog of the Bargmann transform mapping the Fock space $F_{2}\left(\mathbb{C}^{n}\right)$ onto $L_{2}\left(\mathbb{R}^{n}\right)$. For more details on Bargmann transform in connection with time-frequency analysis and Toeplitz-type operators in this context see [14, Section 2].

Section 3: Calderón-Toeplitz operators The representation of wavelet subspaces $A^{(k)}$ is then used to study Toeplitz-type operators based on the Calderón reproducing formula. Given a function (symbol) $a=a(\zeta), \zeta \in G$, the so-called Calderón-Toeplitz operator $T_{a}^{(k)}$ acting on wavelet subspace $A^{(k)}$ is defined as follows

$$
T_{a}^{(k)} f=P^{(k)}(a f), \quad f \in A^{(k)} .
$$

The operators $T_{a}^{(k)}$ naturally arise in connection with localization in time-scale analysis context and they are a filtered version (with $a$ being a filter) of considered signal $f$ analyzed using wavelets $\psi^{(k)}$. The main idea here is to use the constructed unitary operators $R_{k}$ and $R_{k}^{*}$ to reduce the Calderón-Toeplitz operator $T_{a}^{(k)}$ acting on wavelet subspace $A^{(k)}$ to its unitary equivalent image $R_{k} T_{a}^{(k)} R_{k}^{*}$ acting on $L_{2}\left(\mathbb{R}_{+}\right)$. Indeed, the main result of Theorem 3.2 which gives an easy and direct access to the properties of Calderón-Toeplitz operators, then reads as follows:

The Calderón-Toeplitz operator $T_{a}^{(k)}$ with a symbol $a=a(v), v \in \mathbb{R}_{+}$, acting on wavelet subspace $A^{(k)}$ is unitarily equivalent to the multiplication operator $\mathfrak{A}_{a}^{(k)}=$ 
$\gamma_{a, k} I$ acting on $L_{2}\left(\mathbb{R}_{+}\right)$, where the function $\gamma_{a, k}: \mathbb{R}_{+} \rightarrow \mathbb{C}$ has the form

$$
\gamma_{a, k}(\xi)=\int_{\mathbb{R}_{+}} a\left(\frac{v}{2 \xi}\right) \ell_{k}^{2}(v) \mathrm{d} v, \quad \xi \in \mathbb{R}_{+} .
$$

As it can be seen, the function $\gamma_{a, k}$ is obtained by integrating a dilation of a symbol $a=a(v)$ of a Calderón-Toeplitz operator $T_{a}^{(k)}$ against a Laguerre function of order $k$. This result extends the result of Vasilevski for the classical Toeplitz operators acting on the Bergman space (i.e., the case $k=0$ in our notation) in very interesting way which differs from the case of Toeplitz operators acting on weighted Bergman spaces studied in paper [8] and then summarized in Vasilevski book 31. Moreover, in Section 3 we present a number of results following immediately from this equivalency including the spectral-type representation of Calderón-Toeplitz operators whose symbols depend only on imaginary coordinate in the upper half-plane, as well as formulas for the Wick symbols and the star product in terms of our function $\gamma_{a, k}$. In this section we also briefly mention the form of operators $R_{k} T_{a}^{(k)} R_{k}^{*}$ for a symbol a depending only on horizontal variable in the upper half-plane, and for a symbol $a$ in a special and important product form. This presents a way how certain pseudo-differential operators naturally appear in this context of Calderón-Toeplitz operators, and opens the door to many important and interesting questions.

Section 4: Some properties of function $\gamma_{a, k}$ Since the function $\gamma_{a, k}$ given by (11) is responsible for many interesting features and behavior of the corresponding Calderón-Toeplitz operator $T_{a}^{(k)}$ acting on $A^{(k)}$, in this section we investigate its basic properties. We study the limit behavior of higher order derivatives of $\gamma_{a, k}$ for bounded as well as integrable symbols $a$ which provides information about certain interesting properties of $\gamma_{a, k}$. Then we give sufficient conditions for $\gamma_{a, k}$ to be continuous on the whole $[0,+\infty]$ : a question which is closely related to the behavior of a symbol $a=a(v), v \in \mathbb{R}_{+}$, at a neighborhood of points 0 and $+\infty$. More precisely, we prove the following result:

If $a=a(v)$ is a bounded symbol on $\mathbb{R}_{+}$such that the limits $\lim _{v \rightarrow 0} a(v)=a_{0}$ and $\lim _{v \rightarrow+\infty} a(v)=a_{\infty}$ exist, then for each $k \in \mathbb{Z}_{+}$the function $\gamma_{a, k}$ belongs to the algebra $C[0,+\infty]$ of continuous functions on $[0,+\infty]$. Moreover, $\gamma_{a, k}(+\infty)=a_{0}$ and $\gamma_{a, k}(0)=a_{\infty}$ for each $k \in \mathbb{Z}_{+}$.

The result states that the limit at infinity and at zero of the function $\gamma_{a, k}$ is independent of parameter $k$. In fact, it depends only on a limit of the corresponding symbol $a$, but not on the particularly chosen Laguerre functions. This is surprising because the wavelet transforms with Laguerre functions of order $k$ live, up to a multiplier isomorphism, in the so-called true polyanalytic Bergman space of order $k$, which is rather different from the classical Bergman space of analytic functions (corresponding to $k=0$ in our notation). Thus, this result contains the remarkable observation that, asymptotically, all the true polyanalytic Bergman spaces have the same behavior, when such behavior is observed via the association with the Calderón-Toeplitz operator described in Theorem 3.2. This result has some important consequences in quantum physics, signal analysis and in the asymptotic theory of random matrices, which are not yet completely understood. 
Section 5: Isomorphism between the Calderón-Toeplitz operator algebra and functional algebra In connection with the above mentioned results some operator algebras and a functional dependence of Calderón-Toeplitz operators are described in this section. In particular, for any $a(v)=\chi_{[0, \lambda]}(v)$ with $\lambda \in \mathbb{R}_{+}$(here $\chi_{[0, \lambda]}(v)$ is the characteristic function of the interval $[0, \lambda]$ ) we have

$$
\gamma_{\chi_{[0, \lambda]}, k}(\xi)=1-N_{2 k}(2 \lambda \xi) \mathrm{e}^{-2 \lambda \xi}, \quad \xi \in \mathbb{R}_{+},
$$

where $N_{2 k}$ is a certain polynomial of degree $2 k$ (see the interesting result of Lemma 6.2 in Appendix). Thus,

$$
T_{\chi_{[0, \lambda]}^{(k)}}^{(k)} R_{k}^{*} \gamma_{\chi_{[0, \lambda]}, k} R_{k}
$$

and each $C^{*}$-algebra generated by the Calderón-Toeplitz operators $T_{\chi_{[0, \lambda]}^{(k)}}^{(i s}$ isometric and isomorphic to the algebra $C[0,+\infty]$. Moreover, if we consider a symbol $a=a(v)$ from the algebra $L_{\infty}^{\{0,+\infty\}}\left(\mathbb{R}_{+}\right)$of bounded functions on $\mathbb{R}_{+}$ having limits at the endpoints 0 and $+\infty$ such that the corresponding function $\gamma_{a, k}$ separates the points of $[0,+\infty]$, then we construct the function

$$
\nabla_{a, \lambda}^{(k)}(x)=-\frac{1}{\lambda} \ln (1-x) \int_{\mathbb{R}_{+}} a(v)(1-x)^{v / \lambda} L_{k}^{2}\left(-\frac{v}{\lambda} \ln (1-x)\right) \mathrm{d} v
$$

on $[0,1]$ such that each Calderón-Toeplitz operator $T_{a}^{(k)}$ acting on $A^{(k)}$ with a symbol $a=a(v) \in L_{\infty}^{\{0,+\infty\}}\left(\mathbb{R}_{+}\right)$is the function of Toeplitz operator acting on the Bergman space $A^{(0)}=\mathcal{A}^{2}(\Pi)$ with symbol $\chi_{[0, \lambda]}(v)$, i.e.,

$$
T_{a}^{(k)}=\nabla_{a, \lambda}^{(k)}\left(T_{\chi_{[0, \lambda]}^{(0)}}^{(0)}\right.
$$

Interpretation and applicability of these results from the viewpoint of localization in the time-frequency analysis are discussed in the end of Section 5 .

\section{Representation of wavelet subspaces}

In this section we summarize basic methods, constructions and results from our previous works, mainly from $[1]$. We use the obvious notations: $\mathbb{R}(\mathbb{C}, \mathbb{N})$ are the sets of all real (complex, natural) numbers, $\overline{\mathbb{R}}=\mathbb{R} \cup\{-\infty,+\infty\}$ is the twopoint compactification of $\mathbb{R}$, and $\mathbb{R}_{+}\left(\mathbb{R}_{-}\right)$are the positive (negative) half-lines with $\chi_{+}\left(\chi_{-}\right)$their characteristic functions, respectively.

The affine group and admissible wavelets on the real line Let $G=$ $\{\zeta=(u, v) ; u \in \mathbb{R}, v>0\}$ be the locally compact the so-called "ax $+b$ "-group with the group law

$$
(u, v) \diamond\left(u^{\prime}, v^{\prime}\right)=\left(v u^{\prime}+u, v v^{\prime}\right)
$$

and the left-invariant Haar measure $\mathrm{d} \nu(\zeta)=v^{-2} \mathrm{~d} u \mathrm{~d} v$. We identify the group $G$ with the upper half-plane $\Pi=\{\zeta=u+\mathrm{i} v ; u \in \mathbb{R}, v>0\}$ in the complex plane $\mathbb{C}\left(\right.$ with $\mathrm{i}^{2}=-1$ ) equipped with the hyperbolic measure $\mathrm{d} \nu$. Then $L_{2}(G, \mathrm{~d} \nu)$ is the space of all square-integrable functions on $G$ with respect to the measure $\mathrm{d} \nu$. In what follows $\langle\cdot, \cdot\rangle$ always means the inner product on $L_{2}(\mathbb{R})$, whereas 
$\langle\cdot, \cdot\rangle_{G}$ denotes the inner product on $L_{2}(G, \mathrm{~d} \nu)$. For their corresponding norms we use the notation $\|\cdot\|$ and $\|\cdot\|_{G}$, respectively.

A function $\psi \in L_{2}(\mathbb{R})$ is called an admissible wavelet if it satisfies the admissibility condition

$$
\int_{\mathbb{R}_{+}}|\hat{\psi}(x \xi)|^{2} \frac{\mathrm{d} \xi}{\xi}=1
$$

for almost every $x \in \mathbb{R}$, where $\hat{\psi}$ stands for the unitary Fourier transform $\mathcal{F}: L_{2}(\mathbb{R}) \rightarrow L_{2}(\mathbb{R})$ given by

$$
\mathcal{F}\{g\}(\xi)=\hat{g}(\xi)=\int_{\mathbb{R}} g(x) \mathrm{e}^{-2 \pi \mathrm{i} x \xi} \mathrm{d} x .
$$

Let us mention the interesting constructions generalizing admissible wavelets using group-theoretical methods which are given in recent papers of Kisil, see [18] and 19 .

Wavelets related to Laguerre functions In many problems in mathematical physics, signal analysis, parameter identification, analytical and computer studies, etc., the Laguerre functions

$$
\ell_{n}^{(\alpha)}(y)=\left[\frac{n !}{\Gamma(n+\alpha+1)}\right]^{1 / 2} y^{\alpha / 2} \mathrm{e}^{-y / 2} L_{n}^{(\alpha)}(y), \quad y \in \mathbb{R}_{+},
$$

naturally appear. Here, $\Gamma$ is the Euler Gamma function, and $L_{n}^{(\alpha)}$ is the Laguerre polynomial of degree $n \in \mathbb{Z}_{+}=\mathbb{N} \cup\{0\}$ and type $\alpha$ (may be considered as an arbitrary complex number) given by

$$
L_{n}^{(\alpha)}(y)=\frac{y^{-\alpha} \mathrm{e}^{y}}{n !} \frac{d^{n}}{d y^{n}}\left(\mathrm{e}^{-y} y^{n+\alpha}\right)=\sum_{i=0}^{n}(-1)^{i}\left(\begin{array}{c}
n+\alpha \\
n-i
\end{array}\right) \frac{y^{i}}{i !}, \quad y \in \mathbb{R}_{+},
$$

cf. [7, formula 8.970.1]. Recall that the system of functions $\left\{\ell_{n}^{(\alpha)}(y)\right\}_{n \in \mathbb{Z}_{+}}$for $\alpha>-1$ forms an orthonormal basis in the space $L_{2}\left(\mathbb{R}_{+}, \mathrm{d} y\right)$, i.e.,

$$
\int_{\mathbb{R}_{+}} \ell_{m}^{(\alpha)}(y) \ell_{n}^{(\alpha)}(y) \mathrm{d} y=\delta_{m n}, \quad m, n \in \mathbb{Z}_{+} .
$$

For $\alpha=0$ we will simply write $L_{n}(y)$ and $\ell_{n}(y)$. In what follows we proceed as in [11, and for $k \in \mathbb{Z}_{+}$we consider the functions $\psi^{(k)}$ and $\bar{\psi}^{(k)}$ on $\mathbb{R}$ defined on the Fourier transform side as follows

$$
\hat{\psi}^{(k)}(\xi)=\chi_{+}(\xi) \sqrt{2 \xi} \ell_{k}(2 \xi), \quad \text { and } \quad \hat{\bar{\psi}}^{(k)}(\xi)=\hat{\psi}^{(k)}(-\xi),
$$

respectively. Note that Ingrid Daubechies used $\varphi$ with $\hat{\varphi}(\xi)=\sqrt{2 \xi} \hat{\psi}^{(0)}(\xi)$ as the wavelet function in her famous book [5]. It is obvious that for each $k \in \mathbb{Z}_{+}$the functions $\psi^{(k)}$ and $\bar{\psi}^{(k)}$ satisfy the admissibility condition (2). Then according to the Calderón reproducing formula, cf. [3],

$$
f(u)=\int_{\mathbb{R}_{+}}\left(\psi_{v}^{(k)} * \psi_{v}^{(k)} * f\right)(u) \frac{d v}{v^{2}}, \quad g(u)=\int_{\mathbb{R}_{+}}\left(\bar{\psi}_{v}^{(k)} * \bar{\psi}_{v}^{(k)} * g\right)(u) \frac{d v}{v^{2}},
$$




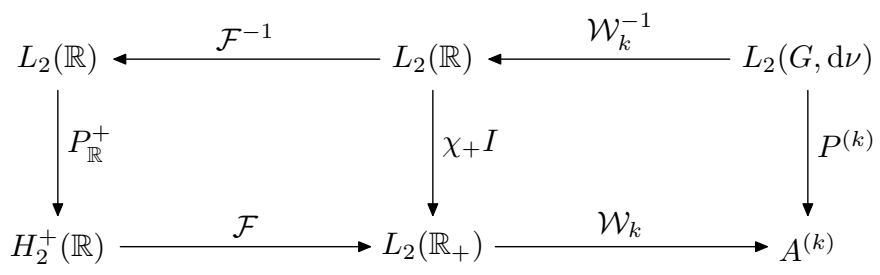

Figure 1: Relationship among the introduced spaces and operators

for all $f \in H_{2}^{+}(\mathbb{R})$ and $g \in H_{2}^{-}(\mathbb{R})$, where $H_{2}^{+}(\mathbb{R})$, resp. $H_{2}^{-}(\mathbb{R})$, are the Hardy spaces, i.e.,

$$
\begin{aligned}
& H_{2}^{+}(\mathbb{R})=\left\{h \in L_{2}(\mathbb{R}) ; \operatorname{supp} \hat{h} \subseteq[0,+\infty)\right\} ; \\
& H_{2}^{-}(\mathbb{R})=\left\{h \in L_{2}(\mathbb{R}) ; \operatorname{supp} \hat{h} \subseteq(-\infty, 0]\right\},
\end{aligned}
$$

respectively. Here $*$ denotes the usual convolution on $L_{2}(\mathbb{R})$, and $\psi_{v}(u)=$ $v^{-1 / 2} \psi(u / v),(u, v) \in G$, is a dilation of $\psi$ on $L_{2}(\mathbb{R})$. It is well-known that $H_{2}^{+}(\mathbb{R})$ and $H_{2}^{-}(\mathbb{R})$ are the only proper invariant subspaces under the (quasiregular) representation $\rho$ of $G$ on $L_{2}(\mathbb{R})$ given by

$$
\left(\rho_{\zeta} f\right)(x)=\frac{1}{\sqrt{v}} f\left(\frac{x-u}{v}\right), \quad \zeta=(u, v) \in G .
$$

Continuous wavelet transform and wavelet subspaces For each $k \in \mathbb{Z}_{+}$ define the subspaces $A^{(k)}$ and $\bar{A}^{(k)}$ of $L_{2}(G, \mathrm{~d} \nu)$ as follows

$$
\begin{aligned}
& A^{(k)}:=\left\{\left(\mathcal{W}_{k} f\right)(u, v)=\left(f * \psi_{v}^{(k)}\right)(u) ; f \in H_{2}^{+}(\mathbb{R})\right\} ; \\
& \bar{A}^{(k)}:=\left\{\left(\mathcal{W}_{\bar{k}} f\right)(u, v)=\left(f * \bar{\psi}_{v}^{(k)}\right)(u) ; f \in H_{2}^{-}(\mathbb{R})\right\},
\end{aligned}
$$

respectively. Note that $\mathcal{W}_{k} f$, resp. $\mathcal{W}_{\bar{k}} f$, are known as the continuous wavelet transforms of functions $f \in H_{2}^{+}(\mathbb{R})$, resp. $f \in H_{2}^{-}(\mathbb{R})$, with respect to wavelets $\psi^{(k)}$, resp. $\bar{\psi}^{(k)}$. Moreover, $\mathcal{W}_{k}$ and $\mathcal{W}_{\bar{k}}$ are isometries from $L_{2}(\mathbb{R})$ to $L_{2}(G, \mathrm{~d} \nu)$ for each $k \in \mathbb{Z}_{+}$. Consequently, $A^{(k)}$, resp. $\bar{A}^{(k)}$, will be called the spaces of Calderón (or wavelet) transforms. We also use the term wavelet subspaces (of $\left.L_{2}(G, \mathrm{~d} \nu)\right)$.

The relationship among the introduced spaces $A^{(k)}$ of wavelet transforms of $H_{2}^{+}(\mathbb{R})$-functions, and the unitary operators of continuous wavelet transform $\mathcal{W}_{k}$ and the Fourier transform $\mathcal{F}$ is schematically described on Figure 1 , Note that $\mathcal{F}^{-1}: L_{2}(\mathbb{R}) \rightarrow L_{2}(\mathbb{R})$ and $\mathcal{W}_{k}^{-1}: A^{(k)} \rightarrow L_{2}(\mathbb{R})$ are the inverse Fourier transform and the inverse continuous wavelet transform, respectively. Observe that Figure 1 also includes the following well-known classical result.

Lemma 2.1 The Fourier transform $\mathcal{F}$ gives an isometrical isomorphism of the space $L_{2}(\mathbb{R})$ onto itself under which

(i) the Hardy space $H_{2}^{+}(\mathbb{R})$, resp. $H_{2}^{-}(\mathbb{R})$, is mapped onto $L_{2}\left(\mathbb{R}_{+}\right)$, resp. $L_{2}\left(\mathbb{R}_{-}\right)$; i.e.,

$$
\mathcal{F}: H_{2}^{+}(\mathbb{R}) \rightarrow L_{2}\left(\mathbb{R}_{+}\right), \quad \text { resp. } \quad \mathcal{F}: H_{2}^{-}(\mathbb{R}) \rightarrow L_{2}\left(\mathbb{R}_{-}\right) ;
$$


(ii) the Szegö projection $P_{\mathbb{R}}^{+}: L_{2}(\mathbb{R}) \rightarrow H_{2}^{+}(\mathbb{R})$, resp. $P_{\mathbb{R}}^{-}: L_{2}(\mathbb{R}) \rightarrow H_{2}^{-}(\mathbb{R})$, is unitarily equivalent to the following one

$$
\mathcal{F} P_{\mathbb{R}}^{+} \mathcal{F}^{-1}=\chi_{+} I, \quad \text { resp. } \quad \mathcal{F} P_{\mathbb{R}}^{-} \mathcal{F}^{-1}=\chi_{-} I .
$$

For each $k \in \mathbb{Z}_{+}$the spaces $A^{(k)}$ and $\bar{A}^{(k)}$ are the reproducing kernel Hilbert spaces. Explicit formulas for their reproducing kernels

$$
K_{\zeta}^{(k)}(\eta)=\left\langle\rho_{\eta} \psi^{(k)}, \rho_{\zeta} \psi^{(k)}\right\rangle, \quad \text { resp. } \quad \bar{K}_{\zeta}^{(k)}(\eta)=\overline{K_{\zeta}^{(k)}(\eta)},
$$

and orthogonal projections $P^{(k)}: L_{2}(G, \mathrm{~d} \nu) \rightarrow A^{(k)}$, resp. $\bar{P}^{(k)}: L_{2}(G, \mathrm{~d} \nu) \rightarrow$ $\bar{A}^{(k)}$, are given in [11]. Note also, that the functions $\psi^{(k)}$ are not normalized in $L_{2}(\mathbb{R})$, since

$$
\left\|\rho_{\zeta} \psi^{(k)}\right\|^{2}=\left\|\psi^{(k)}\right\|^{2}=\left\|\hat{\psi}^{(k)}\right\|^{2}=\frac{1}{2} \int_{\mathbb{R}_{+}} x \ell_{k}^{2}(x) \mathrm{d} x=\frac{2 k+1}{2}:=\kappa_{k},
$$

where the formula

$$
\int_{\mathbb{R}_{+}} x \ell_{k}^{2}(x) \mathrm{d} x=2 k+1, \quad k \in \mathbb{Z}_{+}
$$

has been used (as a special case of formula (15) from Appendix). Therefore, the constant $\kappa_{k}$ appears in formulas of Wick calculus, see Section 3 .

Structural results for wavelet subspaces In what follows we introduce some important operators used in our study, see [11] for more details. Interpret the space $L_{2}(G, \mathrm{~d} \nu)$ as tensor product in the form

$$
L_{2}(G, \mathrm{~d} \nu(\zeta))=L_{2}(\mathbb{R}, \mathrm{d} u) \otimes L_{2}\left(\mathbb{R}_{+}, v^{-2} \mathrm{~d} v\right)
$$

with $\zeta=(u, v) \in G$, and consider the unitary operator

$$
U_{1}=(\mathcal{F} \otimes I): L_{2}(\mathbb{R}, \mathrm{d} u) \otimes L_{2}\left(\mathbb{R}_{+}, v^{-2} \mathrm{~d} v\right) \rightarrow L_{2}(\mathbb{R}, \mathrm{d} \omega) \otimes L_{2}\left(\mathbb{R}_{+}, v^{-2} \mathrm{~d} v\right) .
$$

For the purpose to "linearize" the hyperbolic measure $\mathrm{d} \nu$ onto the usual Lebesgue plane measure we introduce the unitary operator

$$
U_{2}: L_{2}(\mathbb{R}, \mathrm{d} \omega) \otimes L_{2}\left(\mathbb{R}_{+}, v^{-2} \mathrm{~d} v\right) \rightarrow L_{2}(\mathbb{R}, \mathrm{d} x) \otimes L_{2}\left(\mathbb{R}_{+}, \mathrm{d} y\right)
$$

given by

$$
U_{2}: F(\omega, v) \longmapsto \frac{\sqrt{2|x|}}{y} F\left(x, \frac{y}{2|x|}\right) .
$$

Then the inverse operator

$$
U_{2}^{-1}=U_{2}^{*}: L_{2}(\mathbb{R}, \mathrm{d} x) \otimes L_{2}\left(\mathbb{R}_{+}, \mathrm{d} y\right) \rightarrow L_{2}(\mathbb{R}, \mathrm{d} \omega) \otimes L_{2}\left(\mathbb{R}_{+}, v^{-2} \mathrm{~d} v\right)
$$

is given by the rule

$$
U_{2}^{-1}: F(x, y) \longmapsto \sqrt{2|\omega|} v F(\omega, 2|\omega| v) .
$$

Using the classical result of Lemma 2.1 and the operator $U=U_{2} U_{1}$ we get the following theorem describing the structure of wavelet subspaces $A^{(k)}$ and $\bar{A}^{(k)}$ inside $L_{2}(G, \mathrm{~d} \nu)$, see [11, Theorem 2.1] for its proof. 


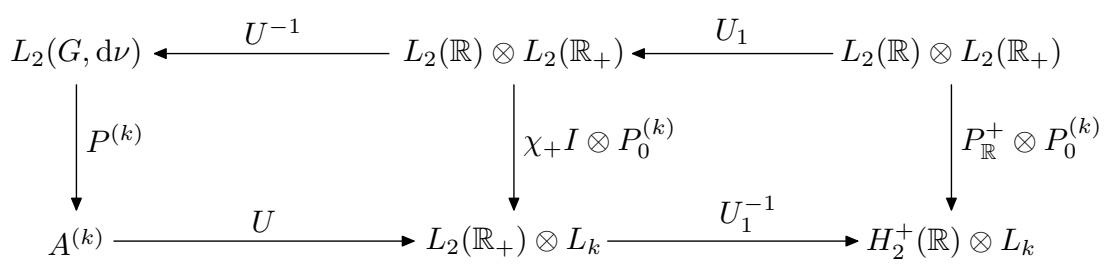

Figure 2: Visualizing the results of Theorem 2.2 and Theorem 2.3

Theorem 2.2 The unitary operator $U=U_{2} U_{1}$ gives an isometrical isomorphism of the space $L_{2}(G, \mathrm{~d} \nu)$ onto $L_{2}(\mathbb{R}, \mathrm{d} x) \otimes L_{2}\left(\mathbb{R}_{+}, \mathrm{d} y\right)$ under which

(i) the space $A^{(k)}$ is mapped onto $L_{2}\left(\mathbb{R}_{+}\right) \otimes L_{k}$, where $L_{k}$ is the rank-one space generated by Laguerre function $\ell_{k}(y)=\mathrm{e}^{-y / 2} L_{k}(y)$;

(ii) the projection $P^{(k)}: L_{2}(G, \mathrm{~d} \nu) \rightarrow A^{(k)}$ is unitarily equivalent to the following one

$$
U P^{(k)} U^{-1}=\chi_{+} I \otimes P_{0}^{(k)},
$$

where $P_{0}^{(k)}$ given by

$$
\left(P_{0}^{(k)} H\right)(y)=\ell_{k}(y) \int_{\mathbb{R}_{+}} H(t) \ell_{k}(t) \mathrm{d} t
$$

is the one-dimensional projection of $L_{2}\left(\mathbb{R}_{+}, \mathrm{d} y\right)$ onto $L_{k}$.

The theorem may be stated analogously for the space $\bar{A}^{(k)}$. Moreover, we may say more about the connection between the wavelet subspaces and Hardy spaces. Indeed, as a direct consequence of Lemma 2.1 and Theorem 2.2 we have

Theorem 2.3 The unitary operator $V=\left(\mathcal{F}^{-1} \otimes I\right) U_{2}(\mathcal{F} \otimes I)$ gives an isometrical isomorphism of the space $L_{2}(G, \mathrm{~d} \nu)$ onto $L_{2}(\mathbb{R}, \mathrm{d} x) \otimes L_{2}\left(\mathbb{R}_{+}, \mathrm{d} y\right)$ under which

(i) the spaces $A^{(k)}$ and $H_{2}^{+}(\mathbb{R})$ are connected by the formula

$$
V\left(A^{(k)}\right)=H_{2}^{+}(\mathbb{R}) \otimes L_{k}
$$

(ii) the projections $P^{(k)}$ and $P_{\mathbb{R}}^{+}$are connected by the formula

$$
V P^{(k)} V^{-1}=P_{\mathbb{R}}^{+} \otimes P_{0}^{(k)} .
$$

The analogous result holds for $\bar{A}^{(k)}, H_{2}^{-}(\mathbb{R})$, and $\bar{P}^{(k)}, P_{\mathbb{R}}^{-}$, respectively. The diagram on Figure 2 schematically describes all the relations among the constructed operators and spaces appearing in the above two theorems. Note that the constructed operators $U_{1}$ and $U_{2}$ may serve also for other purposes, e.g., may be useful in the study of certain operator algebras, but here we will not continue in this direction. 


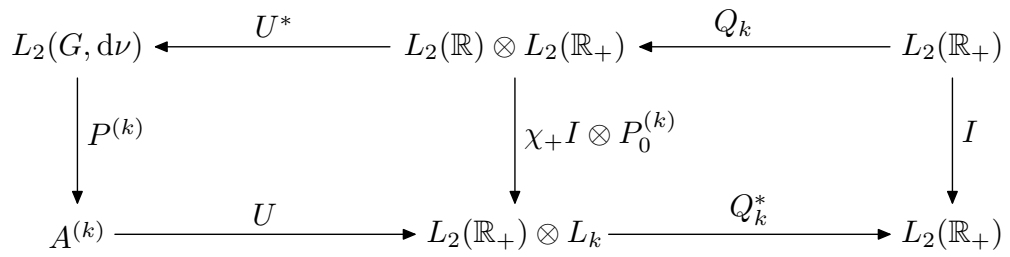

Figure 3: Decomposition of orthogonal projection $P^{(k)}$ and identity $I$ on $L_{2}\left(\mathbb{R}_{+}\right)$

Remark 2.4 The connection between spaces of wavelet transforms (with respect to the specific Bergman wavelet and functions from the Hardy space) and Bergman spaces is well-known, see [10, Theorem 3.1] and references given therein. In fact, this result was a source of motivation for our research in [10 for general wavelets. On the other hand, the connection between the Hardy spaces and poly-Bergman spaces was described in [30, Theorem 4.5]. From this point of view the above results reveal that poly-Bergman spaces and wavelet subspaces share intriguing patterns that may prove usable. For the deeper study of this connection see the paper [1] with some interesting applications to wavelet (super)frames. Moreover, this suggested technique was recently successfully used to obtain a complete characterization of all lattice sampling and interpolating sequences in the Bargmann-Fock space of polyanalytic functions, cf. 2], having a great potential in various applications.

Construction of Bargmann-type transforms For the purpose to construct an exact analog of the Bargmann transform (mapping the Fock space $F_{2}\left(\mathbb{C}^{n}\right)$ onto $L_{2}\left(\mathbb{R}^{n}\right)$ ), first let us introduce the isometric imbedding

$$
Q_{k}: L_{2}\left(\mathbb{R}_{+}\right) \rightarrow L_{2}(\mathbb{R}) \otimes L_{2}\left(\mathbb{R}_{+}\right)
$$

given by

$$
\left(Q_{k} f\right)(x, y)=\chi_{+}(x) f(x) \ell_{k}(y) .
$$

Here the function $f$ is extended to an element of $L_{2}(\mathbb{R})$ by setting $f(x) \equiv 0$ for $x<0$. Its adjoint operator

$$
Q_{k}^{*}: L_{2}(\mathbb{R}) \otimes L_{2}\left(\mathbb{R}_{+}\right) \rightarrow L_{2}\left(\mathbb{R}_{+}\right)
$$

is given by

$$
\left(Q_{k}^{*} F\right)(x)=\chi_{+}(x) \int_{\mathbb{R}_{+}} F(x, t) \ell_{k}(t) \mathrm{d} t .
$$

Then the operator $R_{k}=Q_{k}^{*} U$ maps the space $L_{2}(G, \mathrm{~d} \nu)$ onto $L_{2}\left(\mathbb{R}_{+}\right)$, and the restriction

$$
\left.R_{k}\right|_{A^{(k)}}: A^{(k)} \rightarrow L_{2}\left(\mathbb{R}_{+}\right)
$$

is an isometrical isomorphism. The adjoint operator

$$
R_{k}^{*}=U^{*} Q_{k}: L_{2}\left(\mathbb{R}_{+}\right) \rightarrow A^{(k)} \subset L_{2}(G, \mathrm{~d} \nu)
$$


is an isometrical isomorphism of the space $L_{2}\left(\mathbb{R}_{+}\right)$onto $A^{(k)}$. Clearly, see also Figure 3, operators $R_{k}$ and $R_{k}^{*}$ provide the following decompositions of the projection $P^{(k)}$ and of the identity operator on $L_{2}\left(\mathbb{R}_{+}\right)$, i.e.,

$$
\begin{aligned}
& R_{k} R_{k}^{*}=I: L_{2}\left(\mathbb{R}_{+}\right) \rightarrow L_{2}\left(\mathbb{R}_{+}\right), \\
& R_{k}^{*} R_{k}=P^{(k)}: L_{2}(G, \mathrm{~d} \nu) \rightarrow A^{(k)} .
\end{aligned}
$$

By a direct computation we have the explicit forms of both operators providing Bargmann-type transforms in our situation.

Theorem 2.5 The isometrical isomorphism $R_{k}^{*}=U^{*} Q_{k}: L_{2}\left(\mathbb{R}_{+}\right) \rightarrow A^{(k)}$ is given by

$$
\left(R_{k}^{*} f\right)(\zeta)=\sqrt{2} v \int_{\mathbb{R}_{+}} f(\xi) \ell_{k}(2 \xi v) \mathrm{e}^{2 \pi \mathrm{i} \xi u} \sqrt{\xi} \mathrm{d} \xi,
$$

with $\zeta=(u, v) \in G$. The inverse isomorphism $R_{k}=Q_{k}^{*} U: A^{(k)} \rightarrow L_{2}\left(\mathbb{R}_{+}\right)$has the following form

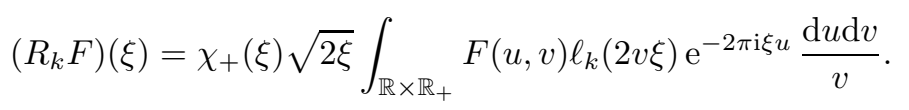

An interesting question how these operators are related to induced representations of the affine group $G$ is solved in recent paper [6].

\section{Calderón-Toeplitz operators}

Wavelet transforms, including the ones coming from the affine group $G$, are the building block of localization operators, see book 33 for further details on wavelet transforms and localization operators. The representation of wavelet subspaces summarized in previous section is especially important in the study of Toeplitz-type operators related to wavelets which symbols depend only on vertical variable $v=\Im \zeta$ in the upper half-plane $\Pi$ of the complex plane $\mathbb{C}$, see [10] for the general setting. This "restriction" to imaginary part of a complex number is due to the decomposition scheme we have just used, but on the other hand, it allows to investigate properties of Toeplitz-type operators in a very elegant way. Moreover, it gives rise to commutative algebras of these operators in both cases of bounded and also unbounded symbols which will be of further interest elsewhere.

For a given bounded function $a$ on $G$ define the Toeplitz-type operator $T_{a}^{(k)}$ : $A^{(k)} \rightarrow A^{(k)}$ with symbol $a$ as

$$
T_{a}^{(k)}=P^{(k)} M_{a}
$$

where $M_{a}$ is the operator of pointwise multiplication by $a$ on $L_{2}(G, \mathrm{~d} \nu)$ and $P^{(k)}$ is the orthogonal projection from $L_{2}(G, \mathrm{~d} \nu)$ onto $A^{(k)}$.

Remark 3.1 In what follows we always consider the operators $T_{a}^{(k)}$ acting on wavelet subspaces $A^{(k)}$ although we may also define the operators $\bar{T}_{a}^{(k)}$ acting on $\bar{A}^{(k)}$ and given by $\bar{T}_{a}^{(k)}=\bar{P}^{(k)} M_{a}$. It is worth noting that in this case of many 
wavelet subspaces (parameterized by $k$ ) other Toeplitz- and Hankel-type (or, Ha-plitz in the terminology of paper [21]) operators may be defined as follows

$$
\begin{aligned}
T_{a}^{(k, l)} & =P^{(k)} M_{a} P^{(l)}, \\
h_{a}^{(k, l)} & =\bar{P}^{(k)} M_{a} P^{(l)}, \\
H_{a}^{(k, l)} & =\left(I-\sum_{j=0}^{k} P^{(j)}\right) M_{a} P^{(l)},
\end{aligned}
$$

see the works [15] and [16].

There exists an alternative way how to get the Calderón-Toeplitz operators. In fact, $T_{a}^{(k)}$ may be viewed as operators acting on $L_{2}(\mathbb{R})$ defined by the formula

$$
\left\langle T_{a}^{(k)} f, g\right\rangle=\int_{G} a(\zeta)\left\langle f, \rho_{\zeta} \psi^{(k)}\right\rangle\left\langle\rho_{\zeta} \psi^{(k)}, g\right\rangle \mathrm{d} \nu(\zeta), \quad f, g \in L_{2}(\mathbb{R}),
$$

interpreted in a weak sense. In this case the identity $T_{1}^{(k)} f=f$ is known as the Calderón reproducing formula, cf. 3, usually being used to define classes of Hilbert spaces with reproducing kernels. Therefore these operators are known as the Calderón-Toeplitz operators, cf. 22, and were introduced by Richard Rochberg in 27] as a wavelet counterpart of Toeplitz operators defined on Hilbert spaces of holomorphic functions. Also, they are an effective timefrequency localization tool in the context of wavelet analysis, see [5], which provides ways of analyzing signals by describing their frequency content as it varies over time, and therefore they are a natural counterpart to the intensively studied localization operators in time-frequency analysis, see e.g. [4. From it follows that the Calderón-Toeplitz operator $T_{a}^{(k)}$ may be viewed as a filtered version of a signal $f$ with a symbol $a$ being considered as a time-varying filter emphasizing or eliminating some kind of information contained in time-scale content on level $k$. For further information and results for Calderón-Toeplitz operators we refer to papers of Nowak 22, 23, 24, and of Rochberg [27, 28, 29 .

Now we will demonstrate the usefulness of Bargmann-type transform $R_{k}$ and its inverse $R_{k}^{*}$ (given by (6) and (5)) under which we may study the unitary equivalent images $R_{k} T_{a}^{(k)} R_{k}^{*}$ of Calderón-Toeplitz operators $T_{a}^{(k)}$, see also the paper [13] for a slightly different approach, but more general results obtained therein. For our investigations the following result is very important because in the case of symbols depending on vertical coordinate of $G$ it enables us to reduce the Calderón-Toeplitz operator to a certain multiplication operator. For the sake of completeness we give its short proof here.

Theorem 3.2 Let $(u, v) \in G$. If a measurable symbol $a=a(v)$ does not depend on u, then the Calderón-Toeplitz operator $T_{a}^{(k)}$ acting on $A^{(k)}$ is unitarily equivalent to the multiplication operator $\mathfrak{A}_{a}^{(k)}=\gamma_{a, k} I$ acting on $L_{2}\left(\mathbb{R}_{+}\right)$, where the function $\gamma_{a, k}: \mathbb{R}_{+} \rightarrow \mathbb{C}$ is given by

$$
\gamma_{a, k}(\xi)=\int_{\mathbb{R}_{+}} a\left(\frac{v}{2 \xi}\right) \ell_{k}^{2}(v) \mathrm{d} v, \quad \xi \in \mathbb{R}_{+} .
$$


Proof. From construction of operators presented in the previous section we directly have the following sequence of operator equalities

$$
\begin{aligned}
\mathfrak{A}_{a}^{(k)} & =R_{k} T_{a}^{(k)} R_{k}^{*}=R_{k} P^{(k)} M_{a} P^{(k)} R_{k}^{*}=R_{k}\left(R_{k}^{*} R_{k}\right) a(v)\left(R_{k}^{*} R_{k}\right) R_{k}^{*} \\
& =\left(R_{k} R_{k}^{*}\right) R_{k} a(v) R_{k}^{*}\left(R_{k} R_{k}^{*}\right)=R_{k} a(v) R_{k}^{*} \\
& =Q_{k}^{*} U_{2} U_{1} a(v) U_{1}^{-1} U_{2}^{-1} Q_{k}=Q_{k}^{*} U_{2} a(v) U_{2}^{-1} Q_{k} .
\end{aligned}
$$

Since for a function $F \in L_{2}(\mathbb{R}, \mathrm{d} x) \otimes L_{2}\left(\mathbb{R}_{+}, \mathrm{d} y\right)$ holds

$$
\left(U_{2} a(v) U_{2}^{-1} F\right)(x, y)=U_{2}(a(v) \sqrt{2|\omega|} v F(\omega, 2|\omega| v))=a\left(\frac{y}{2|x|}\right) F(x, y),
$$

then

$$
\begin{aligned}
\left(\mathfrak{A}_{a}^{(k)} f\right)(\xi) & =\left(Q_{k}^{*} U_{2} a(v) U_{2}^{-1} Q_{k} f\right)(\xi) \\
& =Q_{k}^{*}\left[\chi_{+}(x) a\left(\frac{y}{2|x|}\right) f(x) \ell_{k}(y)\right](\xi) \\
& =f(\xi) \chi_{+}(\xi) \int_{\mathbb{R}_{+}} a\left(\frac{t}{2|\xi|}\right) \ell_{k}^{2}(t) \mathrm{d} t \\
& =\gamma_{a, k}(\xi) f(\xi), \quad \xi \in \mathbb{R}_{+},
\end{aligned}
$$

which completes the proof.

We may observe that the function $\gamma_{a, k}$ is constructed by putting a multiplier in admissibility condition (2) with respect to wavelet $\psi^{(k)}$. As we will see later the function $\gamma_{a, k}$ is responsible for many properties of the corresponding Calderón-Toeplitz operator $T_{a}^{(k)}$ with a symbol $a=a(v)$ (bounded, but also unbounded one), and it shed a new light upon the investigation of properties of the corresponding Calderón-Toeplitz operator.

Let us mention that for general symbols $a=a(u, v)$ on $G$ the CalderónToeplitz operator $T_{a}^{(k)}$ is no longer unitarily equivalent to a multiplication operator. In fact, the operator $R_{k} T_{a}^{(k)} R_{k}^{*}$ may have a more complicated structure: we clarify this statement for a symbol depending only on the first individual coordinate of $G$. This follows from our recent paper [13 as a special case of Theorem 3.8 presented therein, but for the sake of completeness and a little different approach we present here the formal computations providing its proof. In what follows put $\mathbb{R}_{+}^{2}:=\mathbb{R}_{+} \times \mathbb{R}_{+}$.

Theorem 3.3 Let $(u, v) \in G$. If a measurable function $b=b(u)$ does not depend on $v$, then the Calderón-Toeplitz operator $T_{b}^{(k)}$ acting on $A^{(k)}$ is unitarily equivalent to the operator $\mathfrak{B}_{b}^{(k)}$ acting on $L_{2}\left(\mathbb{R}_{+}\right)$given by

$$
\left(\mathfrak{B}_{b}^{(k)} f\right)(\xi)=\int_{\mathbb{R}_{+}} \mathcal{B}_{k}(\xi, t) \hat{b}(\xi-t) f(t) \mathrm{d} t, \quad \xi \in \mathbb{R}_{+},
$$

where the function $\mathcal{B}_{k}: \mathbb{R}_{+}^{2} \rightarrow \mathbb{C}$ has the form

$$
\mathcal{B}_{k}(\xi, t)=\frac{2 \sqrt{t \xi}}{t+\xi} P_{k}\left(\frac{8 t \xi}{(t+\xi)^{2}}-1\right)
$$


with

$$
P_{n}(x)=\frac{1}{2^{n} n !} \frac{\mathrm{d}^{n}}{\mathrm{~d} x^{n}}\left(x^{2}-1\right)^{n}
$$

being the Legendre polynomial of degree $n \in \mathbb{Z}_{+}$for $x \in[-1,1]$.

Proof. Similarly as in the proof of Theorem 3.2 for a function $f \in L_{2}\left(\mathbb{R}_{+}\right)$ we have

$$
\begin{aligned}
\mathfrak{B}_{b}^{(k)} f & =R_{k} T_{b}^{(k)} R_{k}^{*} f=R_{k} b(u) R_{k}^{*} f \\
& =Q_{k}^{*} U_{2}(\mathcal{F} \otimes I) b(u)\left(\mathcal{F}^{-1} \otimes I\right) U_{2}^{-1} Q_{k} f \\
& =Q_{k}^{*} U_{2}(\mathcal{F} \otimes I) b(u)\left(\mathcal{F}^{-1} \otimes I\right)\left(\chi_{+}(\omega) \sqrt{2|\omega|} v f(\omega) \ell_{k}(2|\omega| v)\right) .
\end{aligned}
$$

Using the convolution theorem for Fourier transform we get

$$
\begin{aligned}
\left(\mathfrak{B}_{b}^{(k)} f\right)(\xi) & =Q_{k}^{*} U_{2}\left(\int_{\mathbb{R}} \chi_{+}(t) \sqrt{2|t|} v \hat{b}(\omega-t) f(t) \ell_{k}(2|t| v) \mathrm{d} t\right)(\xi) \\
& =Q_{k}^{*}\left(\int_{\mathbb{R}_{+}} \sqrt{\frac{t}{|x|}} \hat{b}(x-t) f(t) \ell_{k}\left(\frac{t y}{|x|}\right) \mathrm{d} t\right)(\xi) \\
& =\chi_{+}(\xi) \int_{\mathbb{R}_{+}} \sqrt{\frac{t}{|\xi|}} \hat{b}(\xi-t) f(t)\left(\int_{\mathbb{R}_{+}} \ell_{k}(y) \ell_{k}\left(\frac{t y}{|\xi|}\right) \mathrm{d} y\right) \mathrm{d} t \\
& =\chi_{+}(\xi) \int_{\mathbb{R}_{+}} \hat{b}(\xi-t) f(t)\left(\sqrt{t|\xi|} \int_{\mathbb{R}_{+}} \ell_{k}(t \tau) \ell_{k}(|\xi| \tau) \mathrm{d} \tau\right) \mathrm{d} t .
\end{aligned}
$$

Putting

$$
\mathcal{B}_{k}(\xi, t)=\chi_{+}(\xi) \sqrt{t|\xi|} \int_{\mathbb{R}_{+}} \ell_{k}(t \tau) \ell_{k}(|\xi| \tau) \mathrm{d} \tau, \quad t \in \mathbb{R}_{+},
$$

we have the desired result. It remains to show that $\mathcal{B}_{k}$ has the explicit form given by the formula (8). To this end we use [7, formula 7.414.13] to obtain

$$
\begin{aligned}
\mathcal{B}_{k}(\xi, t) & =\sqrt{t \xi} \int_{\mathbb{R}_{+}} \exp \left[-\tau\left(\frac{t+\xi}{2}\right)\right] L_{k}(t \tau) L_{k}(\xi \tau) \mathrm{d} \tau \\
& =\frac{2 \sqrt{t \xi}}{t+\xi} P_{k}\left(\frac{8 t \xi}{(t+\xi)^{2}}-1\right), \quad(t, \xi) \in \mathbb{R}_{+}^{2},
\end{aligned}
$$

and the proof is complete.

As can be seen, the results of Theorem 3.2 and Theorem 3.3 involving Calderón-Toeplitz operators with symbols depending on the individual coordinates of $G$ describe an analogy between the Calderón-Toeplitz operators and the calculus of pseudo-differential operators. We clarify this fact for the case of symbol in the product form. For the more general result see [13, Theorem 3.10].

Theorem 3.4 Let $(u, v) \in G$. If $c(u, v)=a(v) b(u)$ is a measurable symbol, then the Calderón-Toeplitz operator $T_{c}^{(k)}$ acting on $A^{(k)}$ is unitarily equivalent to the pseudo-differential operator $\mathfrak{C}_{\mathfrak{c}}^{(k)}$ acting on $L_{2}\left(\mathbb{R}_{+}\right)$given by the iterated integral

$$
\left(\mathfrak{C}_{\mathfrak{c}}^{(k)} f\right)(\xi)=\int_{\mathbb{R}} d u \int_{\mathbb{R}_{+}} \mathfrak{c}_{k}(\xi, t, u) f(t) \mathrm{e}^{-2 \pi \mathrm{i}(\xi-t) u} \mathrm{~d} t, \quad \xi \in \mathbb{R}_{+},
$$


where its compound (double) symbol $\mathfrak{c}_{k}: \mathbb{R}_{+}^{2} \times \mathbb{R} \rightarrow \mathbb{C}$ has the form

$$
\mathfrak{c}_{k}(\xi, t, u)=2 \sqrt{t \xi} b(u) \int_{\mathbb{R}_{+}} a(v) \ell_{k}(2 v \xi) \ell_{k}(2 v t) \mathrm{d} v .
$$

Remark 3.5 For each $k \in \mathbb{Z}_{+}$the function $\mathcal{C}_{a, k}: \mathbb{R}_{+}^{2} \rightarrow \mathbb{C}$ given in the form of improper parameter-dependent integral

$$
\mathcal{C}_{a, k}(\xi, t)=2 \sqrt{t \xi} \int_{\mathbb{R}_{+}} a(v) \ell_{k}(2 v \xi) \ell_{k}(2 v t) \mathrm{d} v
$$

provides an "extension" of both functions $\gamma_{a, k}$ and $\mathcal{B}_{k}$. Indeed, in the first case $\gamma_{a, k}$ is a restriction of $\mathcal{C}_{a, k}$ to the diagonal, i.e., $\mathcal{C}_{a, k}(\xi, \xi)=\gamma_{a, k}(\xi)$ for each $\xi \in \mathbb{R}_{+}$, and in the second case $\mathcal{B}_{k}$ is a restriction of $\mathcal{C}_{a, k}$ to a constant symbol $a$, i.e., $\mathcal{C}_{1, k}(\xi, t)=\mathcal{B}_{k}(\xi, t)$ for each $(\xi, t) \in \mathbb{R}_{+}^{2}$. Also it can be proved that for a bounded function $a=a(v)$ the function $\mathcal{C}_{a, k}$ is continuous and bounded on $\mathbb{R}_{+}^{2}$ for each $k \in \mathbb{Z}_{+}$.

In what follows we return back to the case of symbols depending on $v \in \mathbb{R}_{+}$ which provides a number of results for properties of Calderón-Toeplitz operators and their algebras as direct corollaries of Theorem 3.2 .

Given a linear subset $\mathcal{A}$ of $L_{\infty}\left(\mathbb{R}_{+}\right)$, for $k \in \mathbb{Z}_{+}$denote by $\mathcal{T}_{k}(\mathcal{A})$ the $C^{*}$ algebra generated by all Calderón-Toeplitz operators $T_{a}^{(k)}$ with symbols $a \in \mathcal{A}$ acting on the wavelet subspace $A^{(k)}$. As a first useful algebra of symbols we introduce the $C^{*}$-algebra $\mathcal{A}_{\infty}$ of all bounded functions on $G$ depending only on $v=\Im \zeta, \zeta \in G$. Then the following result is in the spirit of Vasilevski results, see e.g. [31, Corollary 10.4.10], obtained for Toeplitz operators on (weighted) Bergman spaces. Put $C_{b}\left(\mathbb{R}_{+}\right):=C\left(\mathbb{R}_{+}\right) \cap L_{\infty}\left(\mathbb{R}_{+}\right)$.

Corollary 3.6 Each $C^{*}$-algebra $\mathcal{T}_{k}\left(\mathcal{A}_{\infty}\right), k \in \mathbb{Z}_{+}$, is commutative and is isometrically imbedded to the algebra $C_{b}\left(\mathbb{R}_{+}\right)$. The isomorphic imbedding

$$
\tau_{\infty}^{(k)}: \mathcal{T}_{k}\left(\mathcal{A}_{\infty}\right) \longrightarrow C_{b}\left(\mathbb{R}_{+}\right)
$$

is generated by the following mapping of generators of $\mathcal{T}_{k}\left(\mathcal{A}_{\infty}\right)$

$$
\tau_{\infty}^{(k)}: T_{a}^{(k)} \longmapsto \gamma_{a, k}(\xi)
$$

where $a \in \mathcal{A}_{\infty}$.

Property to be unitarily equivalent to a multiplication operator permits us to describe easily invariant subspaces of algebra $\mathcal{T}_{k}\left(\mathcal{A}_{\infty}\right)$. Note that the following result still holds for any $C^{*}$-algebra generated by bounded Calderón-Toeplitz operators with unbounded (measurable) symbol depending on the imaginary part of complex number. A more detailed study of boundedness of CalderónToeplitz operators with unbounded symbols is done in paper [12.

Corollary 3.7 Each commutative $C^{*}$-algebra $\mathcal{T}_{k}\left(\mathcal{A}_{\infty}\right)$ is reducible. Every invariant subspace $\mathcal{S}_{k}$ of $\mathcal{T}_{k}\left(\mathcal{A}_{\infty}\right)$ is defined by a measurable subset $S_{k} \subset \mathbb{R}_{+}$and has the form

$$
\mathcal{S}_{k}=\left(R_{k}^{*} \chi_{S_{k}} I\right) L_{2}\left(\mathbb{R}_{+}\right) .
$$


Reverting the statement of Theorem 3.2 we get the following spectral-type representation of a Calderón-Toeplitz operator. Its proof goes directly from Theorem 3.2 and Theorem 2.5 .

Corollary 3.8 For $a \in \mathcal{A}_{\infty}$ the Calderón-Toeplitz operator $T_{a}^{(k)}$ acting on $A^{(k)}$ admits the following representation

$$
\left(T_{a}^{(k)} F\right)(\zeta)=\sqrt{2} v \int_{\mathbb{R}_{+}} \gamma_{a, k}(\xi) \ell_{k}(2 v \xi) f(\xi) \mathrm{e}^{2 \pi \mathrm{i} u \xi} \sqrt{\xi} \mathrm{d} \xi
$$

where $\zeta=(u, v) \in G$ and $f(\xi)=\left(R_{k} F\right)(\xi)$.

It may be observed that all the above stated constructions fit perfectly to the general coherent states scheme summarized e.g. in [31, Appendix A] (i.e., wavelets $=$ affine coherent states). Following this scheme the next result gives the form of the Wick symbol of Calderón-Toeplitz operator $T_{a}^{(k)}$ depending on $v=\Im \zeta$. Note that writing the Calderón-Toeplitz operator $T_{a}^{(k)}$ in terms of its Wick symbol yields exactly the spectral-type representation (9). Recall that $\kappa_{k}$ is the constant depending on $k$ given in (4).

Corollary 3.9 Let $\zeta=(u, v) \in G$. Given $a=a(v) \in \mathcal{A}_{\infty}$, the Wick symbol $\widetilde{a}_{k}(\zeta)$ of the Calderón-Toeplitz operator $T_{a}^{(k)}$ depends only on $v$ as well, and has the form

$$
\widetilde{a}_{k}(v)=2 \kappa_{k}^{-1} v^{2} \int_{\mathbb{R}_{+}} \gamma_{a, k}(\xi) \ell_{k}^{2}(2 v \xi) \xi \mathrm{d} \xi .
$$

The corresponding Wick function is given by the formula

$$
\widetilde{a}_{k}(\zeta, \eta)=\frac{2 t v}{K_{\zeta}^{(k)}(\eta)} \int_{\mathbb{R}_{+}} \gamma_{a, k}(\xi) \ell_{k}(2 v \xi) \ell_{k}(2 t \xi) \mathrm{e}^{-2 \pi \mathrm{i} \xi(u-s)} \xi \mathrm{d} \xi,
$$

with $\zeta=(u, v), \eta=(s, t) \in G$.

Remark 3.10 Formula (10) may be interpreted in the following interesting way. For the Calderón-Toeplitz operator $T_{a}^{(k)}$ acting on $A^{(k)}$ with a symbol $a \in \mathcal{A}_{\infty}$ calculate the corresponding function $\gamma_{a, k}(\xi), \xi \in \mathbb{R}_{+}$. Let us introduce the multiplication operator

$$
\left(M_{a}^{(k)} f\right)(x)=\kappa_{k}^{-1 / 2} a(x) f(x), \quad f \in L_{2}\left(\mathbb{R}_{+}\right) .
$$

Take the function $\left(M_{\mathrm{Id}}^{(k)} \gamma_{a, k}\right)(\xi)$, where Id is the identity function, and consider the Calderón-Toeplitz operator on $A^{(k)}$ with symbol $\left(M_{\mathrm{Id}}^{(k)} \gamma_{a, k}\right)(\xi)$. Then the function

$$
\left(M_{\mathrm{Id}}^{(k)} \gamma_{\left(M_{\mathrm{Id}}^{(k)} \gamma_{a, k}\right)(\xi), k}\right)(v)
$$

is nothing but the Wick symbol of the initial Calderón-Toeplitz operator $T_{a}^{(k)}$, i.e.,

$$
\widetilde{a}_{k}(v)=\widetilde{a}_{k}(\zeta, \zeta)=\left(M_{\mathrm{Id}}^{(k)} \gamma_{\left(M_{\mathrm{Id}}^{(k)} \gamma_{a, k}\right)(\xi), k}\right)(v), \quad \zeta=(u, v) \in G
$$


Let us mention the star product $\star$ defining the composition of two Wick symbols $\widetilde{a}_{A}$ and $\widetilde{a}_{B}$ of two operators $A$ and $B$ as the Wick symbol of their composition $A B$, i.e., $\widetilde{a}_{A} \star \widetilde{a}_{B}=\widetilde{a}_{A B}$. The following result gives the formula for the star product of two Calderón-Toeplitz operators in terms of the corresponding function $\gamma$. Again, it is an immediate consequence of Theorem 3.2 and Corollary 3.9

Corollary 3.11 Let $(u, v) \in G$. Let $T_{a}^{(k)}$ and $T_{b}^{(k)}$ be two Calderón-Toeplitz operators acting on $A^{(k)}$ with symbols $a(v)$ and $b(v)$, and let $\widetilde{a}_{k}(v)$ and $\widetilde{b}_{k}(v)$ be their Wick symbols, respectively. Then the Wick symbol $\widetilde{c_{k}}$ of the composition $T_{a}^{(k)} T_{b}^{(k)}$ is given by

$$
\widetilde{c_{k}}(v)=\left(\widetilde{a}_{k} \star \widetilde{b}_{k}\right)(v)=2 \kappa_{k}^{-1} v^{2} \int_{\mathbb{R}_{+}} \gamma_{a, k}(\xi) \gamma_{b, k}(\xi) \ell_{k}^{2}(2 v \xi) \xi \mathrm{d} \xi .
$$

\section{Some properties of function $\gamma_{a, k}$}

In this section we investigate basic properties of function $\gamma_{a, k}: \mathbb{R}_{+} \rightarrow \mathbb{C}$ related to Calderón-Toeplitz operator $T_{a}^{(k)}$ with a symbol $a=a(v)$ in detail. Firstly, let us summarize that for each $k \in \mathbb{Z}_{+}$and each $a \in \mathfrak{A}_{\infty}$ we have $\gamma_{a, k} \in L_{\infty}\left(\mathbb{R}_{+}\right)$ (for more details on this "boundedness topic" and its consequences for CalderónToeplitz operators see the paper [12]). Moreover, in such a case of bounded symbol $a$ the function $\gamma_{a, k}(\xi)$ is also continuous in each finite point $\xi \in \mathbb{R}_{+}$, and thus $\gamma_{a, k} \in C_{b}\left(\mathbb{R}_{+}\right)$- this fact was already used in Corollary 3.6. The continuity, but also other properties of $\gamma_{a, k}$, may be perhaps better seen if we use some formulas for Laguerre polynomials, more precisely [7, formula 8.976.3] and [26, formula (5), p. 209] combined with the exact form of $L_{n}$ given in (3) enable to rewrite the function $\gamma_{a, k}(\xi)$ in the form

$$
\gamma_{a, k}(\xi)=\sum_{i=0}^{k} \sum_{j=0}^{2 k} \sum_{r=0}^{j} c(k, i, j, r)(1-4 \xi)^{2 i-j}(4 \xi)^{j+1} \int_{\mathbb{R}_{+}} a(v) v^{r} \mathrm{e}^{-2 v \xi} \mathrm{d} v,
$$

where

$$
c(k, i, j, r)=\frac{1}{2^{2 k+1}}\left(\begin{array}{c}
2 k-2 i \\
k-i
\end{array}\right)\left(\begin{array}{c}
2 i \\
j
\end{array}\right)\left(\begin{array}{l}
j \\
r
\end{array}\right)\left(\begin{array}{c}
2 i \\
i
\end{array}\right) \frac{(-1)^{r}}{r !} .
$$

Here again might be seen the close connection with the classical Toeplitz operators acting on weighted Bergman spaces (the so called parabolic case), because the last formula resembles the function $\gamma_{a, \lambda}$ obtained in that case by Vasilevski and his collaborators, see [8, formula (2.6)], or [31, formula (10.4.4), p. 254].

Now, we describe the behavior of higher order derivatives of function

$$
\gamma_{a, k}(\xi)=2 \xi \int_{\mathbb{R}_{+}} a(v) \ell_{k}^{2}(2 v \xi) \mathrm{d} v
$$

as $\xi \rightarrow+\infty$. Trivially, for a constant symbol $a$ on $\mathbb{R}_{+}$the function $\gamma_{a, k}$ is constant on $\mathbb{R}_{+}$for each $k \in \mathbb{Z}_{+}$, and therefore all its higher order derivatives are zero. Let us consider a non-constant bounded function $a=a(v)$ on $\mathbb{R}_{+}$. Differentiating $n$-times (with $n \geq 1$ ) we obtain

$$
\frac{\mathrm{d}^{n} \gamma_{a, k}(\xi)}{\mathrm{d} \xi^{n}}=2 n \int_{\mathbb{R}_{+}} a(v) \frac{\mathrm{d}^{n-1}}{\mathrm{~d} \xi^{n-1}} \ell_{k}^{2}(2 v \xi) \mathrm{d} v+2 \xi \int_{\mathbb{R}_{+}} a(v) \frac{\mathrm{d}^{n}}{\mathrm{~d} \xi^{n}} \ell_{k}^{2}(2 v \xi) \mathrm{d} v,
$$


and thus,

$$
\left|\frac{\mathrm{d}^{n} \gamma_{a, k}(\xi)}{\mathrm{d} \xi^{n}}\right| \leq C\left(\frac{n}{\xi} I_{k}^{(n-1)}(\xi)+I_{k}^{(n)}(\xi)\right)
$$

where

$$
I_{k}^{(m)}(\xi):=\int_{\mathbb{R}_{+}}\left|\frac{\mathrm{d}^{m}}{\mathrm{~d} \xi^{m}} \ell_{k}^{2}(2 v \xi)\right| 2 \xi \mathrm{d} v, \quad m \in \mathbb{Z}_{+} .
$$

Using the formula (17) and function $\Lambda$ from Appendix, the function $I_{k}^{(m)}$ may be rewritten as follows

$$
\begin{aligned}
I_{k}^{(m)}(\xi) & =\frac{1}{\xi^{m}} \sum_{i=0}^{m} \sum_{j=0}^{i}\left(\begin{array}{c}
m \\
i
\end{array}\right)\left(\begin{array}{c}
i \\
j
\end{array}\right) \int_{\mathbb{R}_{+}}(2 v \xi)^{m} \mathrm{e}^{-2 v \xi}\left|L_{k-i+j}^{(i-j)}(2 v \xi) L_{k-j}^{(j)}(2 v \xi)\right| 2 \xi \mathrm{d} v \\
& =\frac{1}{\xi^{m}} \sum_{i=0}^{m} \sum_{j=0}^{i}\left(\begin{array}{c}
m \\
i
\end{array}\right)\left(\begin{array}{c}
i \\
j
\end{array}\right) \int_{\mathbb{R}_{+}} \Lambda_{m, k-i+j, k-j}^{(i-j, j)}(x) \mathrm{d} x .
\end{aligned}
$$

Since the last integral is finite, see the formula (20) from Appendix, then $I_{k}^{(m)}(\xi) \rightarrow 0$ as $\xi \rightarrow+\infty$ for each $m \geq 1$ and each $k \in \mathbb{Z}_{+}$. Clearly, in the case $m=0$ we have

$$
I_{k}^{(0)}(\xi)=\int_{\mathbb{R}_{+}} \Lambda_{0, k, k}^{(0,0)}(x) \mathrm{d} x=\int_{\mathbb{R}_{+}} \ell_{k}^{2}(x) \mathrm{d} x=1
$$

for each $k \in \mathbb{Z}_{+}$, and thus $\xi^{-1} I_{k}^{(0)}(\xi) \rightarrow 0$ as $\xi \rightarrow+\infty$. So, for $a=a(v) \in$ $L_{\infty}\left(\mathbb{R}_{+}\right)$we get that for each $n=1,2, \ldots$ holds

$$
\lim _{\xi \rightarrow+\infty} \frac{\mathrm{d}^{n} \gamma_{a, k}(\xi)}{\mathrm{d} \xi^{n}}=0
$$

for each $k \in \mathbb{Z}_{+}$. One can observe that for a non-constant bounded symbol $a$ on $\mathbb{R}_{+}$each derivative of $\gamma_{a, k}(\xi)$ is unbounded near the point $\xi=0$ for each $k \in \mathbb{Z}_{+}$.

In the following theorem we show that the above observation for behavior of higher order derivatives of $\gamma_{a, k}$ holds also if we replace a bounded function $a=a(v)$ by an $L_{1}$-integrable function on $\mathbb{R}_{+}$. The proof requires a number of estimates which are for better readability given in Appendix.

Theorem 4.1 Let $(u, v) \in G$ and $a=a(v) \in L_{1}\left(\mathbb{R}_{+}\right)$be such that $\gamma_{a, k}(\xi) \in$ $L_{\infty}\left(\mathbb{R}_{+}\right)$. Then for each $n=1,2, \ldots$ the equation (12) holds for each $k \in \mathbb{Z}_{+}$.

Proof. Let $n \geq 1$. Then differentiating $n$-times yields (11), and using the formula (17) from Appendix we have

$$
\begin{aligned}
\frac{\mathrm{d}^{n} \gamma_{a, k}(\xi)}{\mathrm{d} \xi^{n}}= & n(-1)^{n-1} \sum_{i=0}^{n-1} \sum_{j=0}^{i}\left(\begin{array}{c}
n-1 \\
i
\end{array}\right)\left(\begin{array}{c}
i \\
j
\end{array}\right) \frac{I_{k, i, j}^{(n-1)}(\xi)}{\xi} \\
& +(-1)^{n} \sum_{i=0}^{n} \sum_{j=0}^{i}\left(\begin{array}{c}
n \\
i
\end{array}\right)\left(\begin{array}{c}
i \\
j
\end{array}\right) I_{k, i, j}^{(n)}(\xi),
\end{aligned}
$$


where

$$
I_{k, i, j}^{(m)}(\xi):=\frac{1}{\xi^{m}} \int_{\mathbb{R}_{+}} a(v)(2 v \xi)^{m} \mathrm{e}^{-2 v \xi} L_{k-i+j}^{(i-j)}(2 v \xi) L_{k-j}^{(j)}(2 v \xi) 2 \xi \mathrm{d} v, \quad m \in \mathbb{Z}_{+} .
$$

Observe that for $n=1$ the first term in (13) is equal to $2 \xi^{-1} \gamma_{a, k}(\xi)$ which tends to 0 as $\xi \rightarrow+\infty$. We now show that also all the above integrals $I_{k, i, j}^{(m)}(\xi)$ for $m \geq 1$ tend to 0 as $\xi \rightarrow+\infty$.

Let $m \geq 1$. For a sufficiently small $\delta>0$ consider the integral

$$
\begin{aligned}
I_{k, i, j}^{(m)}(\xi) & =\frac{1}{\xi^{m}}\left(\int_{0}^{\delta} a(v)(2 v \xi)^{m} \mathrm{e}^{-2 v \xi} L_{k-i+j}^{(i-j)}(2 v \xi) L_{k-j}^{(j)}(2 v \xi) 2 \xi \mathrm{d} v\right. \\
& \left.+\int_{\delta}^{\infty} a(v)(2 v \xi)^{m} \mathrm{e}^{-2 v \xi} L_{k-i+j}^{(i-j)}(2 v \xi) L_{k-j}^{(j)}(2 v \xi) 2 \xi \mathrm{d} v\right) \\
& :=I_{k, i, j, 1}^{(m)}(\xi)+I_{k, i, j, 2}^{(m)}(\xi) .
\end{aligned}
$$

Using (20) from Appendix we have

$$
\begin{aligned}
\left|I_{k, i, j, 1}^{(m)}(\xi)\right| & \leq \sup _{v \in(0, \delta)}|a(v)| \frac{1}{\xi^{m}} \int_{0}^{\delta}(2 v \xi)^{m} \mathrm{e}^{-2 v \xi}\left|L_{k-i+j}^{(i-j)}(2 v \xi) L_{k-j}^{(j)}(2 v \xi)\right| 2 \xi \mathrm{d} v \\
& =\sup _{v \in(0, \delta)}|a(v)| \frac{1}{\xi^{m}} \int_{0}^{2 \delta \xi} \Lambda_{m, k-i+j, k-j}^{(i-j, j)}(x) \mathrm{d} x \\
& \leq \sup _{v \in(0, \delta)}|a(v)| \frac{1}{\xi^{m}} \int_{\mathbb{R}_{+}} \Lambda_{m, k-i+j, k-j}^{(i-j, j)}(x) \mathrm{d} x \\
& \leq \sup _{v \in(0, \delta)}|a(v)| \frac{\operatorname{const}_{m, k-i+j, k-j}^{(i-j, j)}}{\xi^{m}} .
\end{aligned}
$$

Thus for a sufficiently small $\delta$ and sufficiently large $\xi$ we have

$$
\left|I_{k, i, j, 1}^{(m)}(\xi)\right|<\varepsilon .
$$

To estimate $I_{k, i, j, 2}^{(m)}$ we use the formula (19) from Appendix to get

$$
\begin{aligned}
\left|I_{k, i, j, 2}^{(m)}(\xi)\right| \leq & \frac{2}{\xi^{m-1}} \int_{\delta}^{\infty}|a(v)| \Lambda_{m, k-i+j, k-j}^{(i-j, j)}(2 v \xi) \mathrm{d} v \\
\leq & \sum_{r=0}^{k-i+j} \sum_{s=0}^{k-j} \frac{(i-j+1)_{k-i+j-r}}{(k-i+j-r) ! r !} \frac{(j+1)_{k-j-s}}{(k-j-s) ! s !} \\
& \times \frac{2}{\xi^{m-1}} \int_{\delta}^{\infty}|a(v)|(2 v \xi)^{m+r+s} \mathrm{e}^{-2 v \xi} \mathrm{d} v \\
\leq & \sum_{r=0}^{k-i+j} \sum_{s=0}^{k-j} \frac{(i-j+1)_{k-i+j-r}}{(k-i+j-r) ! r !} \frac{(j+1)_{k-j-s}}{(k-j-s) ! s !} \\
& \times \frac{2 \mathrm{e}^{-\delta \xi}}{\xi^{m-1}} \int_{\delta}^{\infty}|a(v)|(2 v \xi)^{m+r+s} \mathrm{e}^{-v \xi} \mathrm{d} v .
\end{aligned}
$$


Using (21) from Appendix we finally have

$$
\begin{aligned}
\left|I_{k, i, j, 2}^{(m)}(\xi)\right| \leq & \sum_{r=0}^{k-i+j} \sum_{s=0}^{k-j} \frac{(i-j+1)_{k-i+j-r}}{(k-i+j-r) ! r !} \frac{(j+1)_{k-j-s}}{(k-j-s) ! s !} \\
& \times\left(\frac{2(m+r+s)}{\mathrm{e}}\right)^{m+r+s} \frac{2 \mathrm{e}^{-\delta \xi}}{\xi^{m-1}}\|a\|_{L_{1}\left(\mathbb{R}_{+}\right)},
\end{aligned}
$$

which yields that for a sufficiently small $\delta$ and sufficiently large $\xi$ we have

$$
\left|I_{k, j, i, 2}^{(m)}(\xi)\right|<\varepsilon,
$$

and therefore $\lim _{\xi \rightarrow+\infty} I_{k, i, j}^{(m)}(\xi)=0$ for all $m \geq 1$. Finally, from the above also follows that all the integrals

$$
J_{k, i, j}^{(m)}(\xi):=\frac{I_{k, i, j}^{(m)}(\xi)}{\xi} \rightarrow 0 \quad \text { as } \quad \xi \rightarrow+\infty
$$

for all $m \geq 1$, which finishes the proof.

As a consequence we immediately have the following corollary.

Corollary 4.2 If $a=a(v) \in L_{1}\left(\mathbb{R}_{+}\right) \cup L_{\infty}\left(\mathbb{R}_{+}\right)$such that $\gamma_{a, k} \in L_{\infty}\left(\mathbb{R}_{+}\right)$, then for each $n=1,2, \ldots$ the equation (12) holds for each $k \in \mathbb{Z}_{+}$.

We have already mentioned that for a bounded symbol $a$ the function $\gamma_{a, k}$ is continuous on $\mathbb{R}_{+}$for each $k \in \mathbb{Z}_{+}$. Now we are interested in sufficient conditions for its continuity on the whole $\overline{\mathbb{R}}_{+}=[0,+\infty]$ which will be useful when studying certain algebras of operators. First observe that for a ,,very large $\xi "$ the function $\ell_{k}^{2}(2 v \xi)$ has a very sharp maximum at the point $v=0$ and thus the major contribution to the integral for $\xi \rightarrow+\infty$ is determined by values of $a(v)$ at a neighborhood of the point 0 . On the other hand, the major contribution for a ,,very small $\xi$ " is determined by values of $a(v)$ at a neighborhood of $+\infty$. In particular, directly from (7) we deduce that if $a(v)$ has limits at the points 0 and $+\infty$, then

$$
\begin{aligned}
\lim _{\xi \rightarrow+\infty} \gamma_{a, k}(\xi) & =\lim _{v \rightarrow 0} a(v), \\
\lim _{\xi \rightarrow 0} \gamma_{a, k}(\xi) & =\lim _{v \rightarrow+\infty} a(v) .
\end{aligned}
$$

Now we do it precisely in the following theorem.

Theorem 4.3 Let $(u, v) \in G$. If $a=a(v) \in L_{\infty}\left(\mathbb{R}_{+}\right)$and the following limits exist

$$
\lim _{v \rightarrow 0} a(v)=a_{0}, \quad \lim _{v \rightarrow+\infty} a(v)=a_{\infty},
$$

then $\gamma_{a, k}(\xi) \in C[0,+\infty]$, and

$$
\gamma_{a, k}(+\infty)=a_{0}, \quad \gamma_{a, k}(0)=a_{\infty}
$$

for each $k \in \mathbb{Z}_{+}$. 
Proof. Let $\xi \rightarrow+\infty$. For a sufficiently small $\delta>0$ represent the function $\gamma_{a, k}(\xi)$ as follows

$$
\gamma_{a, k}(\xi)=2 \xi\left(\int_{0}^{\delta} a(v) \ell_{k}^{2}(2 v \xi) \mathrm{d} v+\int_{\delta}^{\infty} a(v) \ell_{k}^{2}(2 v \xi) \mathrm{d} v\right):=I_{1}(\xi)+I_{2}(\xi) .
$$

Consider the integral $I_{1}$ in the form

$$
I_{1}(\xi)=2 \xi\left(\int_{0}^{\delta} a_{0} \ell_{k}^{2}(2 v \xi) \mathrm{d} v+\int_{0}^{\delta}\left[a(v)-a_{0}\right] \ell_{k}^{2}(2 v \xi) \mathrm{d} v\right):=I_{1,1}(\xi)+I_{1,2}(\xi) .
$$

Then by Lemma 6.2 from Appendix holds

$$
I_{1,1}(\xi)=a_{0} \int_{0}^{\delta} \ell_{k}^{2}(2 v \xi) 2 \xi \mathrm{d} v=a_{0} \int_{0}^{2 \delta \xi} \ell_{k}^{2}(x) \mathrm{d} x=a_{0}+\Phi(\delta, \xi),
$$

where for a sufficiently large $\xi$ we have

$$
|\Phi(\delta, \xi)|<\frac{\varepsilon}{3},
$$

and thus $\left|I_{1,1}(\xi)-a_{0}\right|<\frac{\varepsilon}{3}$. Also,

$$
\left|I_{1,2}(\xi)\right| \leq \sup _{v \in(0, \delta)}\left|a(v)-a_{0}\right| \int_{0}^{\delta} \ell_{k}^{2}(2 v \xi) 2 \xi \mathrm{d} v=\sup _{v \in(0, \delta)}\left|a(v)-a_{0}\right| \int_{0}^{2 \delta \xi} \ell_{k}^{2}(x) \mathrm{d} x,
$$

which again means that for an appropriate choice of $\delta$ and a sufficiently large $\xi$ we have

$$
\left|I_{1,2}(\xi)\right|<\frac{\varepsilon}{3} .
$$

For the last integral $I_{2}$ we use the Hölder integral inequality and Lemma 6.2 from Appendix to get

$$
\begin{aligned}
\left|I_{2}(\xi)\right| & \leq 2 \xi \int_{\delta}^{\infty}|a(v)| \ell_{k}^{2}(2 v \xi) \mathrm{d} v \leq\|a\|_{L_{\infty}\left(\mathbb{R}_{+}\right)} \int_{\delta}^{\infty} \ell_{k}^{2}(2 v \xi) 2 \xi \mathrm{d} v \\
& =\|a\|_{L_{\infty}\left(\mathbb{R}_{+}\right)}\left(1-\int_{0}^{2 \delta \xi} \ell_{k}^{2}(x) \mathrm{d} x\right)=\|a\|_{L_{\infty}\left(\mathbb{R}_{+}\right)} N_{2 k}(2 \delta \xi) \mathrm{e}^{-2 \delta \xi},
\end{aligned}
$$

i.e., for a sufficiently small $\delta$ and sufficiently large $\xi$ we get

$$
\left|I_{2}(\xi)\right|<\frac{\varepsilon}{3} .
$$

Summarizing the above we have that for any $\varepsilon>0$ and an appropriate $\delta>0$ there is $\xi_{0}>0$ such that for each $\xi \geq \xi_{0}$ one has

$$
\left|\gamma_{a, k}(\xi)-a_{0}\right|<\varepsilon,
$$

which proves that $\lim _{\xi \rightarrow+\infty} \gamma_{a, k}(\xi)=a_{0}$. The second limit may be proved similarly. 
Remark 4.4 The result of Theorem4.3, claiming that the limits at infinity and at zero of the function $\gamma_{a, k}$ are independent of parameter $k$, is rather surprising in this case. Since the wavelet transforms with Laguerre functions of order $k$ live on the true polyanalytic Bergman space of order $k$, then from Theorem 4.3 follows that all the true polyanalytic Bergman spaces have asymptotically the "same behavior". The consequences of this observation in various areas of mathematics and physics are not known for us at this moment.

Remark 4.5 Important assumption of Theorem 4.3 is boundedness of symbol $a(v)$. Moreover, as the following example shows, the condition (14) is not necessary even for bounded symbols: if $a(v)=\sin v, v \in \mathbb{R}_{+}$, then

$$
\gamma_{a, 1}(\xi)=2 \xi \int_{\mathbb{R}_{+}} \sin v \mathrm{e}^{-2 v \xi}(1-2 v \xi)^{2} \mathrm{~d} v=\frac{2 \xi\left(1-16 \xi^{2}+48 \xi^{4}\right)}{\left(1+4 \xi^{2}\right)^{3}}, \quad \xi \in \mathbb{R}_{+},
$$

which yields

$$
\lim _{\xi \rightarrow+\infty} \gamma_{a, 1}(\xi)=\lim _{\xi \rightarrow 0} \gamma_{a, 1}(\xi)=0 .
$$

On the other hand, in the following example we give an unbounded symbol $a(v)$ such that the corresponding function $\gamma$. is continuous on $[0,+\infty]$.

Example 4.6 The symbol

$$
a(v)=\frac{1}{\sqrt{v}} \sin \frac{1}{v}, \quad v \in \mathbb{R}_{+},
$$

is clearly unbounded on $\mathbb{R}_{+}$. For $k=1$ (we omit the details of lengthy computation) the corresponding function $\gamma_{a, 1}$ has the form

$$
\gamma_{a, 1}(\xi)=\frac{\sqrt{2 \pi}}{4} \mathrm{e}^{-2 \sqrt{\xi}}\left[(2 \sqrt{\xi}-8 \xi) \frac{\cos 2 \sqrt{\xi}}{2 \sqrt{\xi}}+(3-2 \sqrt{\xi}) \frac{\sin 2 \sqrt{\xi}}{2 \sqrt{\xi}}\right],
$$

for $\xi \in \mathbb{R}_{+}$. Then a direct computation yields

$$
\lim _{\xi \rightarrow 0} \gamma_{a, 1}(\xi)=\sqrt{2 \pi} \text { and } \lim _{\xi \rightarrow+\infty} \gamma_{a, 1}(\xi)=0,
$$

which also means that the function $\gamma_{a, 1}$ is bounded on $\mathbb{R}_{+}$. For $k \geq 2$ it is difficult to get an explicit form of $\gamma_{a, k}$, so in [12] we present a different approach to verify boundedness of $\gamma_{a, k}$, and consequently, boundedness of the corresponding Calderón-Toeplitz operator $T_{a}^{(k)}$ for each $k \in \mathbb{Z}_{+}$.

Example 4.7 The oscillating symbol $a(v)=\mathrm{e}^{2 v \mathrm{i}}$ is continuous at the point $v=0$. Therefore, $\gamma_{a, k}(+\infty)=a(0)=1$ and it is sufficient to investigate the behavior of $\gamma_{a, k}(\xi)$ as $\xi \rightarrow 0$. The explicit form of the corresponding function $\gamma_{a, k}$ is as follows

$$
\begin{aligned}
\gamma_{a, k}(\xi) & =2 \xi \int_{\mathbb{R}_{+}} \mathrm{e}^{-2 v(\xi-\mathrm{i})} L_{k}^{2}(2 v \xi) \mathrm{d} v=\frac{\xi}{\xi-\mathrm{i}} \int_{\mathbb{R}_{+}} \mathrm{e}^{-t} L_{k}^{2}\left(\frac{t \xi}{\xi-\mathrm{i}}\right) \mathrm{d} t \\
& =\frac{(-1)^{k}}{(\xi-\mathrm{i})^{2 k+1}} \sum_{j=0}^{k}(-1)^{j}\left[\left(\begin{array}{c}
k \\
j
\end{array}\right)\right]^{2} \xi^{2 j+1}, \quad \xi \in \mathbb{R}_{+},
\end{aligned}
$$

where [7, formula 7.414.2] has been used. Clearly, $\lim _{\xi \rightarrow 0} \gamma_{a, k}(\xi)=0$, thus $\gamma_{a, k}(\xi) \in$ $C[0,+\infty]$ for each $k \in \mathbb{Z}_{+}$. 


\section{Isomorphism between the Calderón-Toeplitz operator algebra and functional algebra}

In what follows we use the result of Theorem 4.3 to study certain algebras of Calderón-Toeplitz operators. Therefore we denote by $L_{\infty}^{\{0,+\infty\}}\left(\mathbb{R}_{+}\right)$the $C^{*}$ subalgebra of $L_{\infty}\left(\mathbb{R}_{+}\right)$which consists of all functions having limits at the points 0 and $+\infty$.

Remark 5.1 Considering a non-negative real-valued symbol $a$ from the algebra $L_{\infty}^{\{0,+\infty\}}\left(\mathbb{R}_{+}\right)$with a non-zero value $a_{0}$, we get the following interesting property of function $\gamma_{a, k}: \mathbb{R}_{+} \rightarrow \mathbb{R}_{+}$for each $k \in \mathbb{Z}_{+}$in the form

$$
\lim _{\xi \rightarrow \infty} \frac{\gamma_{a, k}(\lambda \xi)}{\gamma_{a, k}(\xi)}=1 \text { for each } \lambda>0
$$

In other words, see [17], the function $\gamma_{a, k}$ is slowly oscillating at infinity. Moreover, in this case $\gamma_{a, k}$ may be represented by

$$
\gamma_{a, k}(\xi)=\sigma_{a, k}(\xi) \exp \left(\int_{1}^{\xi} \omega_{a, k}(t) \frac{d t}{t}\right),
$$

where $\sigma_{a, k}, \omega_{a, k} \in C\left(\mathbb{R}_{+}\right)$such that

$$
\lim _{\xi \rightarrow \infty} \sigma_{a, k}(\xi)=\sigma>0, \quad \lim _{\xi \rightarrow \infty} \omega_{a, k}(\xi)=0 .
$$

Similarly as in the case of algebras $\mathcal{T}_{k}\left(\mathcal{A}_{\infty}\right)$ we get the following result for algebras $\mathcal{T}_{k}\left(L_{\infty}^{\{0,+\infty\}}\left(\mathbb{R}_{+}\right)\right)$.

Theorem 5.2 Each $C^{*}$-algebra $\mathcal{T}_{k}\left(L_{\infty}^{\{0,+\infty\}}\left(\mathbb{R}_{+}\right)\right)$is isomorphic and isometric to $C[0,+\infty]$ and the isometric isomorphism

$$
\tau_{k}: \mathcal{T}_{k}\left(L_{\infty}^{\{0,+\infty\}}\left(\mathbb{R}_{+}\right)\right) \longrightarrow C[0,+\infty]
$$

is generated by the following mapping of generators of $\mathcal{T}_{k}\left(L_{\infty}^{\{0,+\infty\}}\left(\mathbb{R}_{+}\right)\right)$

$$
\tau_{k}: T_{a}^{(k)} \longmapsto \gamma_{a, k}(\xi)
$$

where $a=a(v) \in L_{\infty}^{\{0,+\infty\}}\left(\mathbb{R}_{+}\right)$.

The inclusion

$$
\tau_{k}\left(\mathcal{T}_{k}\left(L_{\infty}^{\{0,+\infty\}}\left(\mathbb{R}_{+}\right)\right)\right) \subseteq C[0,+\infty]
$$

is obvious from Theorem 4.3, whereas the inverse inclusion follows from the next theorem which is a consequence of the Stone-Weierstrass theorem. Given a function $a_{1}(v)$ denote by $L\left(1, a_{1}\right)$ the linear two-dimensional space generated by 1 and the function $a_{1}$. 
Theorem 5.3 Let $a_{1}(v) \in L_{\infty}^{\{0,+\infty\}}\left(\mathbb{R}_{+}\right)$be a real-valued function such that the corresponding function $\gamma_{a_{1}, k}(\xi)$ separates the points of $\overline{\mathbb{R}}_{+}$. Then each $C^{*}$ algebra $\mathcal{T}_{k}\left(L\left(1, a_{1}\right)\right)$ is isomorphic and isometric to $C[0,+\infty]$ and the isometric isomorphism

$$
\tau_{k}: \mathcal{T}_{k}\left(L\left(1, a_{1}\right)\right) \longrightarrow C[0,+\infty]
$$

is generated by the same mapping

$$
\tau_{k}: T_{a}^{(k)} \longmapsto \gamma_{a, k}(\xi)
$$

of generators of the algebra $\mathcal{T}_{k}\left(L\left(1, a_{1}\right)\right)$.

Remark 5.4 As a consequence of this result we have that given a point $\lambda_{0} \in$ $\mathbb{R}_{+}$, then each $C^{*}$-algebra $\mathcal{T}_{k}\left(L\left(1, \chi_{\left[0, \lambda_{0}\right]}\right)\right.$ is isomorphically isometric to $C[0,+\infty]$. In fact, by Theorem 5.3 we need to show that for each $k \in \mathbb{Z}_{+}$the real-valued function

$$
\gamma_{\chi_{\left[0, \lambda_{0}\right]}, k}(\xi)=\chi_{+}(\xi) \int_{\mathbb{R}_{+}} \chi_{\left[0, \lambda_{0}\right]}\left(\frac{v}{2 \xi}\right) \ell_{k}^{2}(v) \mathrm{d} v=\chi_{+}(\xi) \int_{0}^{2 \lambda_{0} \xi} \ell_{k}^{2}(v) \mathrm{d} v
$$

separates the points of $\overline{\mathbb{R}}_{+}$. Instead of this we have, see Appendix, that the function $\int_{0}^{x} \Lambda_{0, k, k}^{(0,0)}(t) \mathrm{d} t$ is strictly increasing.

The previous results motivate the study of piece-wise constant symbols and algebras generated by Calderón-Toeplitz operators with such symbols. But we will continue in the following direction. Consider the Calderón-Toeplitz operator $T_{a_{+}}^{(0)}$ with symbol $a_{+}(v)=\chi_{[0,1 / 2]}(v)$, which is unitarily equivalent to $\gamma_{a_{+}, 0} I$, where

$$
\gamma_{a_{+}, 0}(\xi)=2 \xi \int_{\mathbb{R}_{+}} \chi_{[0,1 / 2]}(v) \mathrm{e}^{-2 v \xi} \mathrm{d} v=1-\mathrm{e}^{-\xi}, \quad \xi \in \overline{\mathbb{R}}_{+} .
$$

This function is continuous on $\overline{\mathbb{R}}_{+}$with values in $[0,1]$, therefore $T_{a_{+}}^{(0)}$ is selfadjoint and $\operatorname{sp} T_{a_{+}}^{(0)}=[0,1]$. Also, the function $\gamma_{a_{+}, 0}$ is strictly increasing and its inverse has the form

$$
\gamma_{a_{+}, 0}^{-1}(x)=\xi(x)=-\ln (1-x), \quad x \in[0,1] .
$$

Thus, for any function $h$ continuous on $[0,1]$ the operator $h\left(T_{a_{+}}^{(0)}\right)$ is well defined according to the standard functional calculus in $C^{*}$-algebras. Now we will exploit the isomorphism between the Calderón-Toeplitz operator algebra and the functional algebra given in Corollary 3.6.

Theorem 5.5 Let $\Delta_{\lambda}$ be a family of functions parameterized by $\lambda \in \mathbb{R}_{+}$and given by

$$
\Delta_{\lambda}(x)=1-(1-x)^{2 \lambda}, \quad x \in[0,1]
$$

Then

$$
\Delta_{\lambda}\left(T_{a_{+}}^{(0)}\right)=T_{\chi_{[0, \lambda]}^{(0)}}^{\left(0 \mathcal{T}_{0}\right.}\left(L\left(1, \chi_{[0, \lambda]}\right)\right)
$$

Remark 5.6 Observe that each function $\Delta_{\lambda}$ is continuous on $[0,1]$ and $\Delta_{\lambda}(0)=$ $0, \Delta_{\lambda}(1)=1$ for each $\lambda \in \mathbb{R}_{+}$. Some particular cases are also interesting: 
$\Delta_{1 / 2}(x) \equiv x$ for all $x \in[0,1]$, whereas for limit values as $\lambda \rightarrow 0$ and $\lambda \rightarrow+\infty$ we have

$$
\Delta_{0}(x) \equiv 0 \quad \text { and } \quad \Delta_{\infty}(x) \equiv 1,
$$

respectively, for all $x \in(0,1)$. These particular cases lead to the equalities

$$
\Delta_{0}\left(T_{a_{+}}^{(0)}\right)=0, \quad \Delta_{1 / 2}\left(T_{a_{+}}^{(0)}\right)=T_{a_{+}}^{(0)}, \quad \Delta_{\infty}\left(T_{a_{+}}^{(0)}\right)=I .
$$

We have chosen the Calderón-Toeplitz operator $T_{a_{+}}^{(0)}$ as the starting operator because in this specific case the equation

$$
x=\gamma_{a_{+}, 0}(\xi)=1-\mathrm{e}^{-\xi}
$$

admits an explicit solution. But we can start from any Calderón-Toeplitz operator $T_{\chi_{[0, \lambda]}^{(0)}}^{(0)}$ with symbol $a(v)=\chi_{[0, \lambda]}(v), \lambda \in \mathbb{R}_{+}$. Indeed, the function $\gamma_{\chi_{[0, \lambda]}, 0}(\xi)$ is strictly increasing which implies that the function $\Delta_{\lambda}:[0,1] \rightarrow[0,1]$ is strictly increasing as well and thus the function $\Delta_{\lambda}^{-1}$ is well defined and continuous on $[0,1]$. Clearly, for $\lambda_{1}, \lambda_{2} \in \mathbb{R}_{+}$we have

$$
\left(\Delta_{\lambda_{2}} \circ \Delta_{\lambda_{1}}^{-1}\right)\left(T_{\chi_{\left[0, \lambda_{1}\right]}}^{(0)}\right)=T_{\chi_{\left[0, \lambda_{2}\right]}}^{(0)},
$$

where $\circ$ is the usual composition of real functions. This means that for any symbol $a=a(v)=\chi_{[0, \lambda]}(v)$ the Calderón-Toeplitz operator $T_{a}^{(0)}$ belongs to the algebra $\mathcal{T}_{0}\left(L\left(1, \chi_{[0, \lambda]}\right)\right)$ and is the function of the operator $T_{a_{+}}^{(0)}$, i.e.,

$$
T_{\chi_{[0, \lambda]}}^{(0)}=\Delta_{\lambda}\left(T_{a_{+}}^{(0)}\right) .
$$

Moreover, Theorem 5.3 and Remark 5.4 imply that each Calderón-Toeplitz operator with $L_{\infty}^{\{0,+\infty\}}\left(\mathbb{R}_{+}\right)$-symbol can be obtained in a similar way.

Theorem 5.7 Let $a=a(v) \in L_{\infty}^{\{0,+\infty\}}\left(\mathbb{R}_{+}\right)$, and for $x \in[0,1]$ put

$$
\nabla_{a, \lambda}^{(k)}(x)=-\frac{1}{\lambda} \ln (1-x) \int_{\mathbb{R}_{+}} a(v)(1-x)^{v / \lambda} L_{k}^{2}\left(-\frac{v}{\lambda} \ln (1-x)\right) \mathrm{d} v,
$$

where $\lambda \in \mathbb{R}_{+}$. Then

$$
\nabla_{a, \lambda}^{(k)}\left(T_{\chi[0, \lambda]}^{(0)}\right)=T_{a}^{(k)}
$$

Proof. By Theorem 3.2 the Calderón-Toeplitz operator $T_{a}^{(k)}$ is unitarily equivalent to $\gamma_{a, k} I$, where

$$
\gamma_{a, k}(\xi)=2 \xi \int_{\mathbb{R}_{+}} a(v) \ell_{k}^{2}(2 v \xi) \mathrm{d} v, \quad \xi \in \overline{\mathbb{R}}_{+} .
$$

Since $x=\gamma_{\chi_{[0, \lambda]}, 0}(\xi)=1-\mathrm{e}^{-2 \lambda \xi}$, substituting $\xi=\xi(x)=-(2 \lambda)^{-1} \ln (1-x)$ we have

$$
\gamma_{a, k}(\xi(x))=-\frac{1}{\lambda} \ln (1-x) \int_{\mathbb{R}_{+}} a(v) \ell_{k}^{2}\left(-\frac{v}{\lambda} \ln (1-x)\right) \mathrm{d} v=\nabla_{a, \lambda}^{(k)}(x),
$$

which completes the proof. 
Interpretations and applications Consider an arbitrary Toeplitz operator acting on the Bergman space $\mathcal{A}^{2}(\Pi)$ with symbol $\chi_{[0, \lambda]}(\Im \zeta)$ where $\zeta=u+$ $\mathrm{i} v \in \Pi$. Using this operator each Calderón-Toeplitz operator with symbol $a=$ $a(v) \in L_{\infty}^{\{0,+\infty\}}\left(\mathbb{R}_{+}\right)$acting on wavelet subspace $A^{(k)}$ is the function of Toeplitz operator. This result is interesting because it enables to change not only symbol from a "nice class" (as it is in the case of classical Toeplitz operators), but also wavelet as will be explained now.

Let $k \in \mathbb{Z}_{+}$. In Section 2 we have denoted by $\mathcal{W}_{k} f$ the continuous wavelet transform of $f \in H_{2}^{+}(\mathbb{R})$ with respect to wavelet $\psi^{(k)}$. Then the value

$$
\left(\mathcal{W}_{k} f\right)(\zeta)=\frac{1}{\sqrt{v}} \int_{\mathbb{R}} f(x) \overline{\psi^{(k)}\left(\frac{x-u}{v}\right)} \mathrm{d} x, \quad \zeta=(u, v) \in G,
$$

is the wavelet coefficient at time $u$ and scale $v$. This integral measures the comparison of the local shape of the signal $f$ and the shape of wavelet $\psi^{(k)}$. Since $\left(\mathcal{W}_{k} f\right)(\zeta) \in A^{(k)}$, we say that this comparison is made "on the level $k$ ". It is well-known that the change of the value of the dilation factor $v$ serves as a mathematical microscope to zoom in and out of the signal whereas localization in time is achieved by selecting $u$. Thus, some time and frequency localization is achieved for each point $\zeta=(u, v)$ in the wavelet half-plane.

It is well-known that $\left(\mathcal{W}_{k} f\right)(\zeta)$ contains enough information to reconstruct the function $f$ on the level $k$. This inverse transform expresses the fact that no information is lost in the transform and we have a representation of the signal $f$ on the level $k$ as a linear superposition of wavelets $\left(\rho_{\zeta} \psi^{(k)}\right)$ with coefficients $\left(\mathcal{W}_{k} f\right)(\zeta)$. In fact, this "perfect reconstruction of signal $f$ on level $k$ " corresponds to the application of Calderón-Toeplitz operator $T_{a}^{(k)}$ with symbol $a(u, v) \equiv 1$ to the signal $f$. Thus, emphasizing or eliminating some information content in wavelet half-plane we get a filtered version of signal $f$ for other choices of symbol $a$. Therefore, these operators are a version of non-stationary (or, time-varying) filters.

Restrict our attention to the case of symbols $a$ depending only on vertical coordinate $v=\Im \zeta, \zeta \in G$, in wavelet half-plane. Since $v$ is a measure of the duration of the event being examined, then the operator $T_{a_{+}}^{(0)}$ gives a reconstruction of a signal on the segment $\Omega_{1 / 2}=\mathbb{R} \times(0,1 / 2]$ and level 0 . By the result of Theorem [5.5 we may obtain any operator $T_{\chi[0, \lambda]}^{(0)}$ (giving a reconstruction of a signal on the segment $\Omega_{\lambda}=\mathbb{R} \times(0, \lambda]$ and level 0$)$ from this operator $T_{a_{+}}^{(0)}$. In fact, from the reconstruction of a signal on the segment $\Omega_{1 / 2}$ and level 0 we may obtain a reconstruction of the same signal on the same level on an arbitrary segment $\Omega_{\lambda}$ using the function $\Delta_{\lambda}$ which is easy to compute.

Even more interesting is the result of Theorem [5.7. If we know the reconstruction of a signal on a segment $\Omega_{\lambda}$ and level 0 , we might get an arbitrary reconstruction of the signal (as its filtered version using a real bounded function $a$ of scale having limits in critical points of boundary of $\mathbb{R}_{+}$such that the corresponding function $\gamma$. separates the points of $\overline{\mathbb{R}}_{+}$) on an arbitrary level $k$ using the function $\nabla_{a, \lambda}^{(k)}$. Theoretically, for the purpose to study localization of a signal in the wavelet half-plane the result of Theorem 5.7 suggests to consider certain "nice" symbols on the first level 0 (indeed, Toeplitz operators on $\mathcal{A}^{2}(\Pi)$ with symbols as characteristic functions of some interval in $\mathbb{R}_{+}$) instead of possibly complicated $L_{\infty}^{\{0,+\infty\}}\left(\mathbb{R}_{+}\right)$-symbols with respect to "different microscope" 
represented by the level $k$. On the other hand, to compute the corresponding function $\nabla_{a, \lambda}^{(k)}$ need not be always easy.

\section{Appendix: functions and integrals containing Laguerre polynomials}

Here we state some necessary formulas and estimations for functions and integrals containing Laguerre polynomials which were used on different places of this article. First we recall the following important integral formula, cf. 32, formula (16), p. 330],

$$
\begin{aligned}
& \int_{\mathbb{R}_{+}} x^{p} \mathrm{e}^{-x} L_{m}^{(\alpha)}(x) L_{n}^{(\beta)}(x) \mathrm{d} x \\
= & \Gamma(p+1) \sum_{i=0}^{\min \{m, n\}}(-1)^{m+n}\left(\begin{array}{c}
p-\alpha \\
m-i
\end{array}\right)\left(\begin{array}{c}
p-\beta \\
n-i
\end{array}\right)\left(\begin{array}{c}
p+i \\
i
\end{array}\right),
\end{aligned}
$$

where $\Re p>-1, \alpha, \beta>-1, m, n \in \mathbb{Z}_{+}$, and

$$
\left(\begin{array}{l}
a \\
b
\end{array}\right)=\frac{\Gamma(a+1)}{\Gamma(b+1) \Gamma(a-b+1)} .
$$

Applying the formula (15) for $p=1, \alpha=\beta=0$ and $m=n=k$ we get the result in formula (4). Also, the formula (15) plays an important role in many areas of research, e.g., in mathematical physics (in the quantum mechanical treatment of the hydrogen atom).

Recall that the derivative of generalized Laguerre polynomial is given by the formula

$$
\frac{d^{r}}{d x^{r}} L_{k}^{(\alpha)}(x)=(-1)^{r} L_{k-r}^{(\alpha+r)}(x),
$$

cf. [7, formula 8.971.2]. Note that for $k<r$ the $r$-th derivative of $L_{k}^{(\alpha)}$ is always zero, and thus we always use the convention $L_{n}^{(\alpha)}(x)=0$ for $n<0$ and arbitrary $\alpha>-1$. The formula (16) together with the Leibniz rule yields the $n$-th derivative of function $\ell_{k}^{2}(2 v \xi)$, i.e.,

$$
\begin{aligned}
\frac{\mathrm{d}^{n}}{\mathrm{~d} \xi^{n}} \ell_{k}^{2}(2 v \xi) & =\sum_{i=0}^{n}\left(\begin{array}{c}
n \\
i
\end{array}\right) \frac{\mathrm{d}^{n-i}}{\mathrm{~d} \xi^{n-i}} \mathrm{e}^{-2 v \xi} \cdot \sum_{j=0}^{i}\left(\begin{array}{l}
i \\
j
\end{array}\right) \frac{\mathrm{d}^{i-j}}{\mathrm{~d} \xi^{i-j}} L_{k}(2 v \xi) \frac{\mathrm{d}^{j}}{\mathrm{~d} \xi^{j}} L_{k}(2 v \xi) \\
& =(-2 v)^{n} \mathrm{e}^{-2 v \xi} \sum_{i=0}^{n} \sum_{j=0}^{i}\left(\begin{array}{c}
n \\
i
\end{array}\right)\left(\begin{array}{c}
i \\
j
\end{array}\right) L_{k-i+j}^{(i-j)}(2 v \xi) L_{k-j}^{(j)}(2 v \xi) .
\end{aligned}
$$

On many places in this paper the estimation of the following (non-negative) function

$$
\Lambda_{p, m, n}^{(\alpha, \beta)}(x):=x^{p} \mathrm{e}^{-x}\left|L_{m}^{(\alpha)}(x) L_{n}^{(\beta)}(x)\right|, \quad x \in \mathbb{R}_{+},
$$

has been used. In our case all parameters $p, m, n, \alpha, \beta \in \mathbb{Z}_{+}$, but some results hold also for $p, \alpha, \beta$ suitable real numbers, even $p$ may be a suitable complex number with $\Re p>-1$. Clearly, for all admissible values of parameters the function

$$
\int_{0}^{x} \Lambda_{p, m, n}^{(\alpha, \beta)}(t) \mathrm{d} t, \quad x \in \mathbb{R}_{+},
$$


is non-negative, continuous, strictly increasing and bounded on $\mathbb{R}_{+}$. For the latter property see (20). For the purpose of boundedness of (18) we need the following result. Recall that

$$
(x)_{n}=\frac{\Gamma(x+n)}{\Gamma(x)}
$$

is the Pochhammer symbol.

Theorem 6.1 ([20]) For $\alpha \geq-\frac{1}{2}, x \geq 0$ and $n \in \mathbb{Z}_{+}$we have

$$
\left|L_{n}^{(\alpha)}(x)\right| \leq \frac{(\alpha+1)_{n}}{n !} \sigma_{n}^{(\alpha)}\left(\mathrm{e}^{x}\right),
$$

where $\sigma_{n}^{(\alpha)}$ are the Cesàro means of the formal series $\sum_{i=0}^{\infty} b_{i}$ given by the formula

$$
\sigma_{n}^{(\alpha)}\left(\sum_{i=0}^{\infty} b_{i}\right):=\frac{n !}{(\alpha+1)_{n}} \sum_{i=0}^{n} \frac{(\alpha+1)_{n-i}}{(n-i) !} b_{i}, \quad \alpha>-1 .
$$

Using this theorem, for each $\alpha, \beta \geq-\frac{1}{2}, p>-1, x \in \mathbb{R}_{+}$and $m, n \in \mathbb{Z}_{+}$we immediately have

$$
\begin{aligned}
\Lambda_{p, m, n}^{(\alpha, \beta)}(x) & \leq \frac{(\alpha+1)_{m}}{m !} \frac{(\beta+1)_{n}}{n !} x^{p} \mathrm{e}^{-x} \sigma_{m}^{(\alpha)}\left(\mathrm{e}^{x}\right) \sigma_{n}^{(\beta)}\left(\mathrm{e}^{x}\right) \\
& =x^{p} \mathrm{e}^{-x} \sum_{i=0}^{m} \frac{(\alpha+1)_{m-i}}{(m-i) ! i !} x^{i} \cdot \sum_{j=0}^{n} \frac{(\beta+1)_{n-j}}{(n-j) ! j !} x^{j} \\
& =\sum_{i=0}^{m} \sum_{j=0}^{n} \frac{(\alpha+1)_{m-i}}{(m-i) ! i !} \frac{(\beta+1)_{n-j}}{(n-j) ! j !} x^{p+i+j} \mathrm{e}^{-x},
\end{aligned}
$$

and thus for its integral holds

$$
\begin{aligned}
\int_{\mathbb{R}_{+}} \Lambda_{p, m, n}^{(\alpha, \beta)}(x) \mathrm{d} x & \leq \sum_{i=0}^{m} \sum_{j=0}^{n} \frac{(\alpha+1)_{m-i}}{(m-i) ! i !} \frac{(\beta+1)_{n-j}}{(n-j) ! j !} \int_{\mathbb{R}_{+}} x^{p+i+j} \mathrm{e}^{-x} \mathrm{~d} x \\
& =\sum_{i=0}^{m} \sum_{j=0}^{n} \frac{(\alpha+1)_{m-i}}{(m-i) ! i !} \frac{(\beta+1)_{n-j}}{(n-j) ! j !} \Gamma(p+i+j+1) \\
& :=\text { const }_{p, m, n}^{(\alpha, \beta)} .
\end{aligned}
$$

The following observation is easy to verify using the classical methods of calculus: for each $p>0, q>0$ and each $x \in \mathbb{R}_{+}$holds

$$
x^{p} \mathrm{e}^{-q x} \leq\left(\frac{p}{\mathrm{e} q}\right)^{p} .
$$

The following result was very useful in proof of Theorem 4.3, It may be of further interest itself, because in some sense it generalizes the incomplete Gamma function to general Laguerre functions. As far as we know it is not included in any literature we have seen, so for the sake of completeness we give its short proof here which is only of computational nature. 
Lemma 6.2 For each $k \in \mathbb{Z}_{+}$and each $x \in \mathbb{R}_{+}$we have

$$
\int_{0}^{x} \Lambda_{0, k, k}^{(0,0)}(t) \mathrm{d} t=\int_{0}^{x} \ell_{k}^{2}(t) \mathrm{d} t=1-N_{2 k}(x) \mathrm{e}^{-x}
$$

where $N_{2 k}(x)$ is a polynomial of $x$ of degree $2 k$.

Proof. Integrating by parts we have

$$
\int_{0}^{x} \Lambda_{0, k, k}^{(0,0)}(t) \mathrm{d} t=\int_{0}^{x} \mathrm{e}^{-t} L_{k}^{2}(t) \mathrm{d} t=1-\mathrm{e}^{-x} L_{k}^{2}(x)-2 \int_{0}^{x} \mathrm{e}^{-t} L_{k}(t) L_{k-1}^{(1)}(t) \mathrm{d} t
$$

where the formula (16) has been used. The last integral has its sense for $k \geq 1$, whereas for $k=0$ disappears and we have immediately the desired result. Using the explicit form of Laguerre polynomials $L_{n}^{(\alpha)}$ given in (3) we get

$$
\int_{0}^{x} \mathrm{e}^{-t} L_{k}(t) L_{k-1}^{(1)}(t) \mathrm{d} t=\sum_{i=0}^{k} \sum_{j=0}^{k-1}(-1)^{i+j}\left(\begin{array}{c}
k \\
i
\end{array}\right)\left(\begin{array}{c}
k \\
j+1
\end{array}\right) \frac{1}{i ! j !} \int_{0}^{x} \mathrm{e}^{-t} t^{i+j} \mathrm{~d} t .
$$

The last integral is, in fact, the incomplete Gamma function $\gamma(1+i+j, x)$ for which the formula

$$
\gamma(1+n, x)=n !\left(1-\mathrm{e}^{-x} \sum_{p=0}^{n} \frac{x^{p}}{p !}\right), \quad n \in \mathbb{Z}_{+},
$$

holds, see [7, formula 8.352.1]. Summarizing the above yields

$$
\begin{gathered}
\int_{0}^{x} \Lambda_{0, k, k}^{(0,0)}(t) \mathrm{d} t=1-2 \sum_{i=0}^{k} \sum_{j=0}^{k-1}(-1)^{i+j}\left(\begin{array}{c}
k \\
i
\end{array}\right)\left(\begin{array}{c}
k \\
j+1
\end{array}\right)\left(\begin{array}{c}
i+j \\
i
\end{array}\right) \\
-\mathrm{e}^{-x}\left(L_{k}^{2}(x)-2 \sum_{i=0}^{k} \sum_{j=0}^{k-1} \sum_{p=0}^{i+j}(-1)^{i+j}\left(\begin{array}{c}
k \\
i
\end{array}\right)\left(\begin{array}{c}
k \\
j+1
\end{array}\right)\left(\begin{array}{c}
i+j \\
i
\end{array}\right) \frac{x^{p}}{p !}\right) .
\end{gathered}
$$

Since the expression in brackets is a polynomial of $x$ of degree $2 k$, it suffices to show that

$$
S(k):=\sum_{i=0}^{k} \sum_{j=0}^{k-1}(-1)^{i+j}\left(\begin{array}{c}
k \\
i
\end{array}\right)\left(\begin{array}{c}
k \\
j+1
\end{array}\right)\left(\begin{array}{c}
i+j \\
i
\end{array}\right)=0
$$

for each $k \geq 1$. But for each $k \geq 1$ we have

$$
S(k)=\sum_{i=0}^{k}(-1)^{i}\left(\begin{array}{c}
k \\
i
\end{array}\right) \cdot \sum_{j=0}^{k-1} \frac{(-1)^{j}}{j !}\left(\begin{array}{c}
k \\
j+1
\end{array}\right)(i+1)_{j}=\sum_{i=0}^{k}(-1)^{i}\left(\begin{array}{c}
k \\
i
\end{array}\right) Q(i),
$$

where $Q(x)$ is a polynomial of degree $k-1$. Then the result from the theory of finite differences yields $S(k)=0$.

Acknowledgements: Author has been on a postdoctoral stay at the Departamento de Matemáticas, CINVESTAV del IPN (México), when writing this 
paper and investigating the topics presented herein. He therefore gratefully acknowledges the hospitality and support of the mathematics department of CINVESTAV on this occasion. Especially, author wishes to thank Professor Nikolai L. Vasilevski for giving him the book [31] and for his postdoctoral guidance and help during his stay in México. Also, autor's thanks go to Professor Nico M. Temme for drawing his attention to the paper [20] with useful boundedness results for Laguerre polynomials. Moreover, author would like to express his deepest gratitude to anonymous referees for their suggestions and interesting observations. Especially, many important consequences of Theorem 4.3 presented in Introduction are due to the anonymous referee, and we appreciate them very well.

\section{References}

[1] L. D. Abreu, Super-wavelets versus poly-Bergman spaces, (preprint 2009) arXiv:0909.4830v1.

[2] L. D. Abreu, Sampling and interpolation in Bargman-Fock spaces of polyanalytic functions, Appl. Comp. Harm. Anal. 29(3) (2010), 287-302.

[3] A. Calderón, Intermediate spaces and interpolation, the complex method, Studia Math. 24 (1964), 113-190.

[4] E. Cordero and K. Gröchenig, Time-frequency analysis of localization operators, J. Funct. Anal. 205(1) (2003), 107-131.

[5] I. Daubechies, Ten Lectures on Wavelets, SIAM, Philadelphia, PA, 1992.

[6] A. S. Elmabrok, O. Hutník and V. V. Kisil, Induced representations of the affine group and intertwining operators (in preparation).

[7] I. S. Gradshteyn and I. M. Ryzhik, Tables of Integrals, Series and Products, rth Edition, Academic Press, Elsevier, San Diego, 2007.

[8] S. Grudsky, A. Karapetyans and N. Vasilevski, Dynamics of properties of Toeplitz operators on the upper half-plane: parabolic case, J. Operator Theory 52 (2004), 1-31.

[9] O. Hutník, On the structure of the space of wavelet transforms, C. R. Acad. Sci. Paris, Ser. I 346 (2008), 649-652.

[10] O. Hutník, On Toeplitz-type operators related to wavelets, Integral Equations Operator Theory 63(1) (2009), 29-46.

[11] O. Hutník, A note on wavelet subspaces, Monatsh. Math. 160(1) (2010), 59-72.

[12] O. Hutník, On boundedness of Calderón-Toeplitz operators, Integral Equations Operator Theory (to appear), DOI 10.1007/s00020-011-1883-2.

[13] O. Hutník and M. Hutníková, On Toeplitz localization operators, Arch. Math. (to appear). 
[14] M. Hutníková and O. Hutník, An alternative description of Gabor spaces and Gabor-Toeplitz operators, Rep. Math. Phys. 66(2) (2010), 237-250.

[15] Q. Jiang and L. Peng, Toeplitz and Hankel type operators on the upper half-plane, Integral Equations Operator Theory 15 (1992), 744-767.

[16] Q. Jiang and L. Peng, Wavelet transform and Toeplitz-Hankel type operators, Math. Scand. 70 (1992), 247-264.

[17] J. Karamata, Sur un mode de croissance régulière des fonctions, Mathematica (Cluj) 4 (1930), 38-53.

[18] V. V. Kisil, Wavelets beyond admissibility, In: Progress in Analysis and Its Applications - Proceedings of the 7th International ISAAC Congress, Ed. Ruzhansky, M. and Wirth, J., World Scientific, 219-225, 2010.

[19] V. V. Kisil, Covariant transform, J. Phys.: Conf. Series 284 (2011) 012038.

[20] Z. Lewandowski and J. Szynal, An upper bound for the Laguerre polynomials, J. Comput. Appl. Math. 99 (1998), 529-533.

[21] N. K. Nikol'ski, Ha-plitz operators: A survey of some recent results, In: Operators and Function Spaces, Ed. Power, S. C., Reidel, Dordrecht, 87137, 1985.

[22] K. Nowak, On Calderón-Toeplitz operators, Monatsh. Math. 116 (1993), 49-72.

[23] K. Nowak, Commutators based on the Calderón reproducing formula, Studia Math. 104 (1993), 285-306.

[24] K. Nowak, Local Toeplitz operators based on wavelets: phase space patterns for rough wavelets, Studia Math. 119 (1996), 37-64.

[25] R. Quiroga-Barranco and N. L. Vasilevski, Commutative $C^{*}$-algebras of Toeplitz operators on the unit ball, I. Bargmann-type transforms and spectral representations of Toeplitz operators, Integral Equations Operator Theory 59(3) (2007), 379-419.

[26] E. D. Rainville, Special Functions, New York: Macmillan, 1960.

[27] R. Rochberg, Toeplitz and Hankel operators, wavelets, NWO sequences, and almost diagonalization of operators, In: Proc. Symp. Pure Math. 51, part I., Ed. Arveson, W. B. and Douglas, R. G., Amer. Math. Soc, Providence, 425-444, 1990.

[28] R. Rochberg, A correspondence principle for Toeplitz and CalderónToeplitz operators, In: Israel Math. Conf. Proc. 5, Ed. Cwikel, M. et al., Bar-Ilan Univ., Ramat Gan, 229-243, 1992.

[29] R. Rochberg, Eigenvalue estimates for Calderón-Toeplitz operators, In: Lecture Notes in Pure and Appl. Math. 136, Ed. Jarosz, K., Dekker, New York, 345-357, 1992.

[30] N. L. Vasilevski, On the structure of Bergman and poly-Bergman spaces, Integral Equations Operator Theory 33 (1999), 471-488. 
[31] N. L. Vasilevski, Commutative Algebras of Toeplitz Operators on the Bergman Space, Series: Operator Theory: Advances and Applications, Vol. 185, Basel: Birkhäuser, 2008.

[32] Z. X. Wang and D. R. Guo, Special Functions, Singapore: World Scientfic, 1989.

[33] M. W. Wong, Wavelet Transforms and Localization Operators, Series: Operator Theory: Advances and Applications, Vol. 136, Basel: Birkhäuser, 2002.

Ondrej Hutník, Departamento de Matemáticas, CINVESTAV del IPN, Current address: Apartado Postal 14-740, 07000, México, D.F., México

E-mail address: hutnik@math.cinvestav.mx

AND

Institute of Mathematics, Faculty of Science, Pavol Jozef Šafárik University in Košice, Jesenná 5, 04001 Košice, Slovakia,

E-mail address: ondrej.hutnik@upjs.sk 